\title{
Endoatmospheric Ascent Optimal Guidance with Analytical Nonlinear Trajectory Prediction
}

\author{
Shilei Zhao $\mathbb{D}$, Wanchun Chen $(\mathbb{D}$, and Liang Yang $(\mathbb{D}$ \\ School of Astronautics, Beihang University, Xueyuan Road No. 37, Haidian District, Beijing, China \\ Correspondence should be addressed to Liang Yang; yangliang.buaa@hotmail.com
}

Received 11 October 2021; Accepted 23 December 2021; Published 18 January 2022

Academic Editor: Xingling Shao

Copyright (C) 2022 Shilei Zhao et al. This is an open access article distributed under the Creative Commons Attribution License, which permits unrestricted use, distribution, and reproduction in any medium, provided the original work is properly cited.

\begin{abstract}
In this paper, an endoatmospheric ascent optimal guidance law with terminal constraint is proposed, which is under the framework of predictor-corrector algorithm. Firstly, a precise analytical nonlinear trajectory prediction with arbitrary Angle of Attack (AOA) profile is derived. This derivation process is divided into two steps. The first step is to derive the analytical trajectory with zero AOA using a regular perturbation method. The other step is to employ pseudospectral collocation scheme and regular perturbation method to solve the increment equation so as to derive the analytical solution with arbitrary AOA profile. The increment equation is formulated by Taylor expansion around the trajectory with zero AOA which remains the second order increment terms. Therefore, the resulting analytical solutions are the nonlinear functions of high order terms of arbitrary AOA values discretized in Chebyshev-Gauss-Legendre points, which has high accuracy. Secondly, an iterative correction scheme using analytical gradient is proposed to solve the endoatmospheric ascent optimal guidance problem, in which the dynamical constraint is enforced by the resulting analytical solutions. It only takes a fraction of a second to get the guidance command. Nominal simulations, Monte Carlo simulations, and optimality verification are carried out to test the performance of the proposed guidance law. The results show that it not only performs well in providing the optimal guidance command, but also has great applicability, high guidance accuracy and computational efficiency. Moreover, it has great robustness even in large dispersions and uncertainties.
\end{abstract}

\section{Introduction}

For rocket-powered launch vehicles, the guidance accuracy of ascent phase has a significant effect on the flight performance in subsequent missions [1]. In this phase, it involves strong nonlinearity and coupling dynamics resulting from the complicated forces such as thrust, aerodynamic forces, and gravity. That brings some troublesome but intriguing challenges for the ascent guidance design if the terminal trajectory constraint should be enforced strictly. Plenty of efforts have been made to this research field.

Optimal control is widely used for ascent guidance and trajectory tracking [2-4]. The majority methods can be divided into two categories: indirect method and direct method [5]. The indirect method gets the optimal solution by solving two-point boundary-value problem (TPBVP). In [6], Lu et al. proposed a closed-loop endoatmospheric ascent guidance law by using the classical finite difference method for solving TPBVP. Further research and development on improving this method are referred to [7-9]. In [10], $\mathrm{Lu}$ et al. presented a numerical trajectory reconstruction algorithm based on a finite element method, which satisfies the real-time requirement of generating the guidance commands. In $[11,12]$, Dukeman et al. proposed an endoatmospheric ascent guidance for rocket-powered launch vehicles, in which multiple shooting method is employed to solving TPBVP with a high computational efficiency. In addition, many optimization algorithms such as convex optimization [13] and swarming algorithms [14-16] are also used to solve TPBVP resulting from ascent guidance problem. But the indirect method needs to derive the complicated necessary conditions including adjoint equations, transversality conditions, and maximum principle. And it is sensitive to the initial guess, which leads to it being unsuitable for onboard guidance. The direct method transforms the optimal control 
problem into a nonlinear programming problem (NLP), and then well-developed numerical algorithms can be used to solve it in a discrete manner. In $[17,18]$, Ghose et al. adopted a differential evolution method to obtain the optimal ascent phase trajectory for the hypersonic vehicle. In recent years, benefiting from the high accuracy in both the primal and dual solutions and the equivalence between the direct and indirect forms, the pseudospectral method has been widely used in trajectory optimization and guidance design for the ascent phase [19-27]. However, it requires the third-party software to solve the complex NLP problem, which has huge codes and occupies large computational sources. Therefore, it is not suitable for onboard application. In order to alleviate the computing burden of online guidance, several other methods have been applied. The predictor-corrector method has great potential for ascent phase guidance. In [28], Li et al. proposed a novel segmented and weighted adaptive predictor-corrector guidance method for the ascent phase of hypersonic vehicle. In [29], Yang et al. proposed a linear Gauss pseudospectral model predictive control for solving the nonlinear optimal control problem without solving NLP, which has high computational efficiency. It has been successfully applied into entry guidance for the hypersonic vehicle [30-32].

However, all of the mentioned methods heavily rely on numerical integration prediction, which leads to the heavy computation and time-consuming. On the contrary, if the trajectory prediction can be provided in an analytical manner, the computational efficiency can be significantly improved. For the gliding trajectory of hypersonic vehicle, the analytical solution has been widely studied and applied to the entry guidance [33-38]. But there is no study on precise analytical solution to ascent trajectory. The existing ascent guidance laws in an analytic manner ignore the aerodynamic force, which results in large prediction error as for the endoatmospheric trajectory $[39,40]$. In endoatmospheric ascent phase, the variations in velocity, altitude, and flight-path angle are much great, which leads to the great difference in a flight environment. It also involves the controlled thrust and aerodynamics force which results that its dynamics has strong state coupling and nonlinearity. Therefore, it is difficult to find the analytical solution using traditional method.

This paper aims at developing an efficient optimal ascent guidance law which considers multiple constraints and quadratic performance index. This method is under the framework of predictor-corrector algorithm. Firstly, an analytical trajectory prediction with high accuracy is proposed using a regular perturbation method. The solving process consists of two steps. In the first step, the analytical trajectory with zero Angle of Attack (AOA) is derived. In the derivation process, an assumptive constant axial force is introduced to decompose the dynamics, and therefore, the resulting subdynamics is uncoupled and can be solved by integrating analytically. In the second step, Taylor expansion around the analytical trajectory with zero AOA is used to formulate the increment dynamic equation which fully considers the second increment terms. And then, pseudospectral collocation scheme and regular perturbation method are employed to derive the analytical solution of the terminal state, which is a nonlinear function of high order terms of arbitrary AOA values discretized in Chebyshev-GaussLegendre points. Secondly, by introducing a quadric performance index, an endoatmospheric ascent optimal guidance problem is formulated, in which the dynamical constraint is enforced by the resulting analytical solutions. It should be noted that it is impossible to derive the analytical solution to such problem because the high order terms are involved. Then, an iterative solving scheme using analytical gradient is proposed to solve it. This method is attractive from the point of high efficiency, high accuracy with few points, and character that the optimal guidance command for nonlinear guidance problem is provided. The most difference from the previous methods is that the analytical prediction is involved to remove the trajectory integration, which significantly improves the computational efficiency. Furthermore, the high order increment terms are fully considered to bring the high accuracy for analytical prediction. Finally, the accuracy verification for analytical solution, several nominal simulations, comparison with the optimal solution, and Monte Carlo simulations have been carried out to evaluate the performance of the proposed method. The results show that the analytical solutions are highly consistent with the numerical solutions. The proposed method not only performs well in providing the optimal guidance command but also has high computational efficiency and guidance accuracy. Moreover, it has great robustness even in large dispersions and uncertainties.

The remainder of this paper is organized as follows: Section 2 establishes the formulation of ascent phase guidance. Section 3 derives the analytical solution to ascent trajectory. Section 4 provides the optimal AOA to eliminate the terminal errors. And then, the implementation of ascent guidance law using optimal analytical correction is presented in Section 5. Finally, Section 6 provides some simulations to evaluate the performance of the proposed guidance law.

\section{Problem Formulation}

2.1. Ascent Dynamics. During the ascent phase for a pointmass vehicle model, the equations of motion for the vehicle over the nonrotating spherical Earth in the longitudinal plane are established as

$$
\left\{\begin{array}{l}
\dot{V}=\frac{P \cos \alpha-D}{m}-g \sin \gamma \\
\dot{\gamma}=\frac{P \sin \alpha+L}{m V}+\left(\frac{V}{r}-\frac{g}{V}\right) \cos \gamma \\
\dot{h}=V \sin \gamma
\end{array}\right.
$$

where $m, V, \gamma, h$, and $\alpha$ are the mass, velocity, flight-path angle, altitude, and $\mathrm{AOA}$, respectively; $r=R_{e}+h$ is the distance from the center of Earth to the vehicle, where $R_{e}$ is the average radius of the Earth, and $g=\mu / r^{2}$ is the 
gravitational acceleration; $P$ is the thrust of the vehicle; and $L, D$ are the aerodynamic drag and lift expressed as

$$
\begin{aligned}
& L=\frac{1}{2} \rho V^{2} S C_{l}, \\
& D=\frac{1}{2} \rho V^{2} S C_{d},
\end{aligned}
$$

where $\rho$ is the atmospheric density and $S$ is the reference area. The terms, $C_{l}$ and $C_{d}$, are the lift and drag coefficients which are dependent on Mach number and AOA.

2.2. Terminal Constraints and Performance Index. In order to guarantee the successful handover with the subsequent phase, some terminal state, mainly flight-path angle and altitude, must be enforced to the desired ones in the ascent phase. For simplification, the terminal constraints are represented in a general form as

$$
\psi\left(x\left(t_{f}\right)\right)=\left\{\begin{array}{l}
\gamma_{f}-\gamma_{f c}=0, \\
h_{f}-h_{f c}=0,
\end{array}\right.
$$

where $\gamma_{f}$ and $h_{f}$ are the ascent phase terminal flight-path angle and altitude and $\gamma_{f c}$ and $h_{f c}$ are the desired ones, respectively.

For the ascent phase guidance, the control variable is AOA, which determines the direction of the thrust. And the autopilot is used to track the AOA profile to achieve the flight mission. In order to provide the stable input, the weighted squared sum of AOA is selected as the performance index.

$$
J=\frac{1}{2} \int_{t_{0}}^{t_{f}} \frac{\alpha^{2}}{\left(t_{f}-t\right)^{n}} d t .
$$

It is noted that the denominator, $\left(t_{f}-t\right)^{n}$, is used to shape the AOA profile and makes the terminal AOA zero if $n>0$ is selected.

2.3. Vehicle Description. For rocket-powered launch vehicle, its thrust can be formulated as

$$
P=I_{\mathrm{sp}} q_{m} g_{0}-S_{e} P_{a}
$$

where $I_{\text {sp }}$ is specific impulse and $q_{m}$ is the mass flow which can be treated as a constant for the solid launch vehicle; $S_{e}$ is the area of nozzle; and $P_{a}$ is the atmospheric pressure at the flight altitude. Noted that, although the thrust is related to altitude, the change resulting from the altitude is very small. Additionally, the lift and drag coefficients can be fitted to be linear function and parabolic function of AOA due to the axisymmetric aerodynamic configuration, which are formulated as

$$
\begin{aligned}
& C_{l}=C_{l}^{\alpha} \alpha, \\
& C_{d}=C_{d 0}+C_{d}^{\alpha} \alpha^{2},
\end{aligned}
$$

where $C_{l}^{\alpha}, C_{d 0}$, and $C_{d}^{\alpha}$ are all related to Mach number, which can be fitted as the polynomial of Mach number, i.e.,

$$
\begin{aligned}
C_{l}^{\alpha} & =\sum_{i=0}^{p} C_{l, i}^{\alpha} M a^{i}, \\
C_{d 0} & =\sum_{i=0}^{p} C_{d 0, i} M a^{i}, \\
C_{d}^{\alpha} & =\sum_{i=0}^{p} C_{d, i}^{\alpha} M a^{i} .
\end{aligned}
$$

In the solving of the analytical solutions, these coefficients are regarded as the constants, $\bar{C}_{l}^{\alpha}, \bar{C}_{d 0}$, and $\bar{C}_{d}^{\alpha}$, which are the average values during the flight. Because the aerodynamic coefficients do not vary greatly with the Mach when the vehicle is flying at supersonic speed, the error caused by this simplified hypothesis is small. The second stage of a two-stage launch vehicle model, the parameters of which listed in Table 1, is used for all simulations.

\section{Analytical Ascent-Trajectory Prediction}

In this section, the analytical ascent trajectory prediction is developed. Firstly, a simplified condition, in which the AOA is set to zero, is considered. Under the simplification, the analytical solution to ascent trajectory is only related to the flight time for the given initial values and vehicle parameters. Next, based on the presented analytical solution with zero AOA, the analytical solution to trajectory increment corresponding to nonzero AOA is derived. Then, the analytical solution with arbitrary AOA profile, which is the sum of analytical solution with zero AOA and trajectory increment corresponding to nonzero AOA, is provided. In the derivation of these analytical solutions, the regular perturbation method is adopted to decompose the coupled dynamics into a Taylor series which can be solved sequentially.

3.1. Dynamics for Analytical Trajectory. In order to simplify the solutions, the index atmospheric density model, $\rho=$ $\rho_{0} \exp (-\beta h)$, where $\rho_{0}=1.225 \mathrm{~kg} / \mathrm{m}^{3}$ is the sea-level atmospheric density and $\beta=1.389 \times 10^{-4} \mathrm{~m}^{-1}$, is adopted for the solving of the analytical solutions.

In order to avoid confusion, the variables corresponding to zero AOA are expressed with subscript " $b$." For the case of zero AOA, it is obvious that $\bar{P} \sin \alpha+L=0$ and $D=$ $\rho_{b} V_{b}^{2} S C_{0} / 2$; then, the dynamics is simplified to

$$
\left\{\begin{array}{l}
\dot{V}_{b}=\frac{1}{m}\left(\bar{P}-\frac{\rho_{b} V_{b}^{2} S \bar{C}_{d 0}}{2}\right)-g_{b} \sin \gamma_{b}, \\
\dot{\gamma}_{b}=\left(\frac{V_{b}}{r_{b}}-\frac{g_{b}}{V_{b}}\right) \cos \gamma_{b}, \\
\dot{h}_{b}=V_{b} \sin \gamma_{b} .
\end{array}\right.
$$


Table 1: Parameter of the vehicle model.

\begin{tabular}{lccc}
\hline Parameter & Value & Parameter & Value \\
\hline$C_{l, i}^{\alpha}$ & {$[3.774,0.451,-0.075,4.98 e-03,-1.19 e-04]$} & $m_{0}(\mathrm{~kg})$ & 46680 \\
$C_{d 0, i}$ & {$[0.846,-0.152,1.58 e-03,-5.67 e-04]$} & $q_{m}(\mathrm{~kg} / \mathrm{s})$ & 479.9 \\
$C_{d, i}^{\alpha}$ & {$[4.963,0.598,-0.097,7.12 e-03,-1.93 e-04]$} & $I_{\mathrm{sp}}(\mathrm{kN})$ & 264.7 \\
$S\left(\mathrm{~m}^{2}\right)$ & 4.3 & $t_{f}(\mathrm{~s})$ & 60 \\
\hline
\end{tabular}

For simplifying the derivation, a variable related to $\gamma_{b}$ is introduced.

$$
\theta_{b}=\ln \left[\frac{\left(1+\sin \gamma_{b}\right)}{\left(1-\sin \gamma_{b}\right)}\right]
$$

Replacing $\gamma_{b}$ with $\theta_{b}$ as the state variable, the equation of motion presented in (8) can be rewritten as

$$
\left\{\begin{array}{l}
\dot{V}_{b}=\frac{1}{m}\left(\bar{P}-\frac{\rho_{b} V_{b}^{2} S \bar{C}_{d 0}}{2}\right)-g_{b} \sin \gamma_{b} \\
\dot{\theta}_{b}=2\left(\frac{V_{b}}{r_{b}}-\frac{g_{b}}{V_{b}}\right) \\
\dot{h}_{b}=V_{b} \sin \gamma_{b}
\end{array}\right.
$$

The increment of the velocity, flight-path angle, and altitude with nonzero AOA relative to that with zero AOA are defined as $\Delta V, \Delta \gamma$, and $\Delta h$, which are used to derive the analytical solutions with arbitrary AOA profile. It is obvious that the differential equations of those trajectory increments are the difference of (1) and (8). For the sake of simplification, the following Taylor's expansions are adopted.

$$
\left\{\begin{array}{l}
\exp (-\beta \Delta h) \approx 1-\beta \Delta h, \quad \sin \alpha \approx \alpha \\
\frac{1}{V} \approx \frac{1}{V_{b}}-\frac{\Delta V}{V_{b}^{2}}, \quad \cos \alpha \approx 1-\frac{\alpha^{2}}{2} \\
\sin \gamma \approx \sin \gamma_{b}+\cos \gamma_{b} \Delta \gamma-\sin \gamma_{b} \frac{\Delta \gamma^{2}}{2} \\
\cos \gamma \approx \cos \gamma_{b}-\sin \gamma_{b} \Delta \gamma-\cos \gamma_{b} \frac{\Delta \gamma^{2}}{2}
\end{array}\right.
$$

Then, the dynamics equations of increments are established as

$$
\left\{\begin{array}{l}
\Delta \dot{V}=-\frac{1}{2 m}\left[\left(\bar{P}+\rho_{b} V_{b}^{2} S \bar{C}_{d}^{\alpha}\right) \alpha^{2}+2 \rho_{b} V_{b} S C_{d} \Delta V-\beta \rho_{b} V_{b}^{2} S C_{d} \Delta h\right]-g_{i} \cos \gamma_{b} \Delta \gamma+\frac{1}{2} g_{i} \sin \gamma_{b} \Delta \gamma^{2} \\
\Delta \dot{\gamma}=\frac{1}{m}\left[\frac{\bar{P}}{V_{b}}+\frac{1}{2} \rho_{b} V_{b} S \bar{C}_{l}^{\alpha}(1-\beta \Delta h)\right] \alpha+\left(\frac{1}{r_{0}}+\frac{g_{i}}{V_{b}^{2}}\right) \cos \gamma_{b} \Delta V-\left(\frac{V_{b}}{r_{0}}-\frac{g_{i}}{V_{b}}\right)\left(\sin \gamma_{b}+0.5 \cos \gamma_{b} \Delta \gamma\right) \Delta \gamma \\
\Delta \dot{h}=\left(\sin \gamma_{b}+\cos \gamma_{b} \Delta \gamma\right) \Delta V+V_{b}\left(\cos \gamma_{b} \Delta \gamma-\sin \gamma_{b} \frac{\Delta \gamma^{2}}{2}\right)
\end{array}\right.
$$

Because $r \gg \Delta h$, the altitude increment on the value of $1 / r$ is ignored. It should be noted that the second order terms related to the AOA and state increment are remained to achieve high accuracy.

3.2. Analytical Ascent Trajectory with Zero AOA. In (10), the right side of the differential equation of $V_{b}$ can be divided into the following two parts:

$$
\dot{V}_{b}=\frac{F_{\text {ave }}}{m}+\frac{F_{\varepsilon}}{m}
$$

where $F_{\text {ave }}$ is the major axial force, which is the sum of thrust, average atmospheric drag, and average axial component of gravity, and $F_{\varepsilon}$ is the perturbation axial force:

$$
\begin{aligned}
F_{\mathrm{ave}}= & \bar{P}-\frac{\rho_{\mathrm{ave}} V_{\mathrm{ave}}^{2} S \bar{C}_{d 0}}{2}-m_{\mathrm{ave}} g_{\mathrm{ave}} \sin \gamma_{\mathrm{ave}}, \\
F_{\varepsilon}= & \frac{\rho_{\mathrm{ave}} V_{\mathrm{ave}}^{2} S \bar{C}_{d 0}}{2}+m_{\mathrm{ave}} g_{\mathrm{ave}} \sin \gamma_{\mathrm{ave}} \\
& -\frac{\rho_{b} V_{b}^{2} S \bar{C}_{d 0}}{2}-m g_{b} \sin \gamma_{b},
\end{aligned}
$$

where $m_{\text {ave }}, \gamma_{\text {ave }}, V_{\text {ave }}, \rho_{\text {ave }}$, and $g_{\text {ave }}$ are the average mass, flight-path angle, velocity, atmospheric density, and gravitational acceleration, respectively, which can be previously 
determined by the average of its initial and terminal values. In the boost phase, the thrust is usually much greater than the change of aerodynamic drag and the axial component of gravity. Thus, the perturbation axial force is small enough and can be regarded as the correction terms. On the other hand, the trigonometric term $\sin \gamma_{b}$ can be written as follows:

$\sin \gamma_{b}=\sin \gamma_{0}+c_{1} \Delta \theta_{b}+\left(\sin \gamma_{b}-\sin \gamma_{0}-c_{1} \Delta \theta_{b}\right), \quad \Delta \theta_{b}=\theta_{b}-\theta_{0}$,

where $c_{1}$ is the constant coefficient, which can be obtained by the initial and terminal values of $\gamma_{b}$ and $\theta_{b}$ :

$$
c_{1}=\frac{\sin \gamma_{b f}-\sin \gamma_{0}}{\theta_{b f}-\theta_{0}} \text {. }
$$

In the boost phase, the flight-path angle varies slightly; therefore, the linear term $\sin \gamma_{0}+c_{1} \Delta \theta_{b}$ is much larger than the rest terms. Therefore, according to the regular perturbation method presented in Appendix A, (10) can be modified as

$$
\left[\begin{array}{c}
\dot{V}_{b} \\
\dot{\theta}_{b} \\
\dot{h}_{b}
\end{array}\right]=\left[\begin{array}{c}
\frac{F_{\mathrm{ave}}}{m} \\
2\left(\frac{V_{b}}{r_{b}}-\frac{g_{b}}{V_{b}}\right) \\
V_{b}\left(\sin \gamma_{0}+c_{1} \Delta \theta_{b}\right)
\end{array}\right]
$$

$$
+\frac{\varepsilon}{k}\left[\begin{array}{c}
\frac{F_{\varepsilon}}{m} \\
0 \\
V_{b}\left(\sin \gamma_{b}-\sin \gamma_{0}-c_{1} \Delta \theta_{b}\right)
\end{array}\right] \text {, }
$$

where $\varepsilon$ is a small parameter for the subsequent regular perturbation order and $k$ is a constant, which is set to be equal to $\varepsilon$. According to (A.5), the zeroth-order dynamics is

$$
\left\{\begin{array}{l}
\dot{V}_{b}^{(0)}=\frac{F_{\text {ave }}}{m}, \\
\dot{\theta}_{b}^{(0)}=2\left(\frac{V_{b}^{(0)}}{r_{0}}-\frac{g_{i}}{V_{b}^{(0)}}\right), \\
\dot{h}_{b}^{(0)}=V_{b}^{(0)}\left(\sin \gamma_{0}+c_{1} \Delta \theta_{b}^{(0)}\right) .
\end{array}\right.
$$

It is noted that, in order to decouple the differential equations of $\theta_{b}^{(0)}$ and $h_{b}^{(0)}$, the terms related to $r$ are expanded at initial point since the change of $r$ is much smaller than its initial value, $r_{0}$. The first-order dynamics is

$$
\left\{\begin{array}{l}
\dot{V}_{b}^{(1)}=\frac{1}{m}\left[\bar{P}-F_{\mathrm{ave}}-\frac{1}{2} \rho_{0} \exp \left(-\beta h_{b}^{(0)}\right)\left(V_{b}^{(0)}\right)^{2} S \bar{C}_{d 0}\right]-g_{\mathrm{ave}}\left(\sin \gamma_{0}+c_{1} \Delta \theta_{b}^{(0)}\right), \\
\dot{\theta}_{b}^{(1)}=-2\left(\frac{V_{b}^{(0)}}{r_{0}}-\frac{2 g_{i}}{V_{b}^{(0)}}\right) \frac{\Delta h_{b}^{(0)}}{r_{0}}+2\left(\frac{V_{b}^{(0)}}{r_{0}}+\frac{g_{i}}{V_{b}^{(0)}}\right) \frac{V_{b}^{(1)}}{V_{b}^{(0)}}, \\
\dot{h}_{b}^{(1)}=V_{b}^{(0)}\left(\sin \gamma_{b}^{(0)}-\sin \gamma_{0}-c_{1} \Delta \theta_{b}^{(0)}\right)+V_{b}^{(1)} \sin \gamma_{b}^{(0)}+\frac{V_{b}^{(0)} \cos ^{2} \gamma_{b}^{(0)} \theta_{b}^{(1)}}{2},
\end{array}\right.
$$

where $\gamma_{b}^{(0)}$ is the zeroth-order solution to flight-path angle, which can be derived from $\theta_{b}^{(0)}$.

$$
\gamma_{b}^{(0)}=\sin ^{-1}\left\{\frac{\exp \left(\theta_{b}^{(0)}\right)-1}{\exp \left(\theta_{b}^{(0)}\right)+1}\right\} .
$$

It should be noted that the terms related to $h_{b}^{(1)}$ in the differential equations of $\theta_{b}^{(1)}$ are ignored due to $h_{b}^{(1)}$ $\ll \Delta h_{b}^{(0)}$. Additionally, according to the boundary condi- tions, the initial values of zeroth-order and first-order states are

$$
\begin{aligned}
& V_{b 0}^{(0)}=V_{0}, \theta_{b 0}^{(0)}=\theta_{0}, h_{b 0}^{(0)}=h_{0}, \\
& V_{b 0}^{(1)}=0, \theta_{b 0}^{(1)}=0, h_{b 0}^{(1)}=0 .
\end{aligned}
$$

3.2.1. Zeroth-Order Solution. It is obvious that the three differential equations in (18) are not coupled, so that the analytical solution to $V_{b}^{(0)}, \theta_{b}^{(0)}$, and $h_{b}^{(0)}$ can be derived by integrating these three differential equations in sequence. 
Integrating the first equation of (18) from $t_{0}$ to $t$, the analytical solution of $V_{b}^{(0)}$ at time $t$ can be easily obtained as

$$
V_{b}^{(0)}=V_{0}-V_{\mathrm{e}} \ln \left(1-\frac{t-t_{0}}{T}\right),
$$

where $T=m_{0} / q_{\mathrm{m}}$ and $V_{\mathrm{e}}=F_{\text {ave }} / q_{\mathrm{m}}$. Obviously, the differential equations of other variables are all related to $V_{b}^{(0)}$; thus, the analytical solutions of these variables are the functions of $V_{b}^{(0)}$. For the sake of simplifying the expression, a dimensionless variable, $\bar{V}=V_{b}^{(0)} / V_{e}$, is employed, which is regarded as an independent variable in the subsequent derivation. From (22), the expression of $\bar{V}$ is

$$
\bar{V}=\bar{V}_{0}-\ln \left(1-\frac{t-t_{0}}{T}\right)
$$

where $\bar{V}_{0}=V_{0} / V_{e}$ is initial value of $\bar{V}$. Substituting (22) into the first equation of (18), the differential equation of $\bar{V}$ can be expressed as a simple function.

$$
\dot{\bar{V}}=\frac{F_{\text {ave }}}{m V_{e}}=\frac{\exp \left(\bar{V}-\bar{V}_{0}\right)}{T} .
$$
yields

Dividing the second and third equations of (18) by (24)

$$
\frac{d \theta_{b}^{(0)}}{d \bar{V}}=2\left(K_{1} \bar{V}-\frac{K_{2}}{\bar{V}}\right) \exp (-\bar{V})
$$

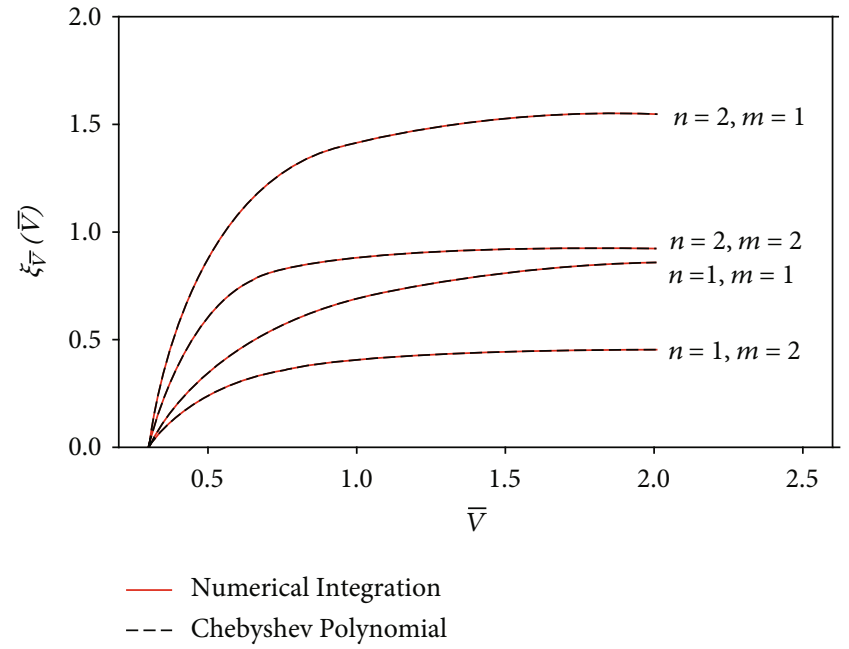

Figure 1: Comparison of $\xi_{\bar{V}}(\bar{V})$ with various $n$ and $m$.

$$
\frac{d h_{b}^{(0)}}{d \bar{V}}=K_{1} r_{0}\left(\sin \gamma_{0} \bar{V}+c_{1} \bar{V} \Delta \theta_{b}^{(0)}\right) \exp (-\bar{V})
$$

where $\quad K_{1}=T V_{\mathrm{e}} \exp \left(\bar{V}_{0}\right) / r_{0}$ and $\quad K_{2}=g_{\mathrm{i}} T \exp \left(\bar{V}_{0}\right) / V_{\mathrm{e}}$. Integrating (25) from $\bar{V}_{0}$ to $\bar{V}, \theta_{b}^{(0)}$ can be obtained as

$$
\theta_{b}^{(0)}(\bar{V})=\theta_{0}+2 K_{1} f_{\bar{V}}(\bar{V} ; 1,1)-2 K_{2} \xi_{\bar{V}}(\bar{V} ; 1,1)
$$

where $f_{\bar{V}}(\bar{V} ; n, m)(n \geq 0, m \geq 0)$ is

$$
f_{\bar{V}}(\bar{V} ; n, m)=\left\{\begin{array}{l}
\frac{\bar{V}^{n+1}-\bar{V}_{0}^{n+1}}{n+1}, \quad m=0, \\
\frac{n !}{m^{n+1}} \sum_{k=0}^{n} \frac{m^{k}}{k !}\left[\bar{V}_{0}^{k} \exp \left(-m \bar{V}_{0}\right)-\bar{V}^{k} \exp (-m \bar{V})\right], \quad m>0 .
\end{array}\right.
$$

$\xi_{\bar{V}}(\bar{V} ; n, m)(n>0, m>0)$ is the integral term expressed as

$$
\xi_{\bar{V}}(\bar{V} ; n, m)=\int_{\bar{V}_{0}}^{\bar{V}} \frac{\exp \left(-m x_{\bar{V}}\right)}{x_{\bar{V}}^{n}} d x_{\bar{V}}
$$

Although the integral term is quite simple, it has no analytical solution. In order to derive its approximate analytical solution, Chebyshev interpolation polynomial is used to approximate the integrand. Let

$$
\frac{\exp \left(-m x_{\bar{V}}\right)}{x_{\bar{V}}^{n}} \approx \sum_{i=0}^{N} \frac{\exp \left(-m x_{\bar{V} i}\right)}{x_{\bar{V} i}^{n}} L_{i}(x)=\sum_{i=0}^{N} p_{\bar{V} i}^{(n, m)} x^{i},
$$

where $x_{\bar{V} i}$ is the value of $\bar{V}$ at the $i$-th interpolation point $x_{i} ; L_{i}(x)(i=0, \cdots, N)$ is the interpolation basis function; and $x=\left(x_{\bar{V}}-\bar{V}_{2}\right) / \bar{V}_{1}$ is the dimensionless variable with $[-1,1]$, where $\bar{V}_{1}=\left(\bar{V}-\bar{V}_{0}\right) / 2$ and $\bar{V}_{2}=\left(\bar{V}+\bar{V}_{0}\right) / 2$. Then, $\xi_{\bar{V}}$ can be approximated as

$$
\xi_{\bar{V}}(\bar{V} ; n, m)=\frac{1}{2}\left(\bar{V}-\bar{V}_{0}\right) \sum_{i=0}^{N} W_{i}(x) \frac{\exp \left(-m x_{\bar{V} i}\right)}{x_{\bar{V} i}^{n}}
$$

where $W_{i}(x)$ is the integral weight coefficient. The derivation process is presented in Appendix B in detail. The error analysis is carried out by comparing the solution obtained by numerical integration and the 6th-degree interpolation polynomials in Figure 1, from which we can see that the interpolation polynomials have high accuracy. 
In order to derive the analytical solution to $h_{b}^{(0)}$, an integral related to $\Delta \theta_{b}^{(0)}$ is introduced.

$I_{\theta}\left(x_{\bar{V}} ; n, m\right)=\int_{\bar{V}_{0}}^{x_{\bar{V}}} \Delta \theta_{b}^{(0)} x_{\bar{V}}^{n} \exp \left(-m x_{\bar{V}}\right) d x_{\bar{V}}, \quad n \geq 0, m>0$.

Using the formula of partial integration, the analytical solution to $I_{\theta}\left(x_{\bar{V}} ; n, m\right)$ is expressed as

$$
\begin{aligned}
I_{\theta}\left(x_{\bar{V}} ; n, m\right)= & -\frac{n !}{m^{n+1}} \sum_{k=0}^{n} \frac{m^{k}}{k !} \\
& \cdot\left[\Delta \theta_{b}^{(0)}\left(x_{\bar{V}}\right) x_{\bar{V}}^{k} \exp \left(-m x_{\bar{V}}\right)-\theta_{r}\left(x_{\bar{V}} ; k, m\right)\right],
\end{aligned}
$$

where $\theta_{r}\left(x_{\bar{V}} ; n, m\right)$ is the integral shown below:

$$
\theta_{r}\left(x_{\bar{V}} ; n, m\right)=\int_{\theta_{0}}^{\theta_{b}^{(0)}} x_{\bar{V}}^{n} \exp \left(-m x_{\bar{V}}\right) d \theta_{b}^{(0)} ; n \geq 0, m>0 .
$$

It is obvious that the analytical solution to $\theta_{r}$ is similar to that of $\theta_{b}^{(0)}$. Substituting (25) into (34), $\theta_{r}$ can be expressed as

$$
\begin{aligned}
\theta_{r}\left(x_{\bar{V}} ; n, m\right) & =2 \int_{\bar{V}_{0}}^{x_{\bar{V}}}\left\{K_{1} x_{\bar{V}}^{n+1} \exp \left[-(m+1) x_{\bar{V}}\right]-K_{2} x_{\bar{V}}^{n-1} \exp \left[-(m+1) x_{\bar{V}}\right]\right\} d x_{\bar{V}} \\
& =\left\{\begin{array}{l}
2 K_{1} f_{\bar{V}}\left(x_{\bar{V}} ; 1, m+1\right)-2 K_{2} \xi_{\bar{V}}\left(x_{\bar{V}} ; 1, m+1\right), \quad n=0, \\
2 K_{1} f_{\bar{V}}\left(x_{\bar{V}} ; n+1, m+1\right)-2 K_{2} f_{\bar{V}}\left(x_{\bar{V}} ; n-1, m+1\right), \quad n>0 .
\end{array}\right.
\end{aligned}
$$

Then, integrating (26) from $\bar{V}_{0}$ to $\bar{V}$, the analytical solution to $h_{b}^{(0)}$ is

$$
h_{b}^{(0)}(\bar{V})=h_{0}+K_{1} r_{0}\left[\sin \gamma_{0} f_{\bar{V}}(\bar{V} ; 1,1)+c_{1} I_{\theta}(\bar{V} ; 1,1)\right]
$$

where

$$
\begin{aligned}
I_{\theta}(\bar{V} ; 1,1)= & 2 K_{1}\left[f_{\bar{V}}(\bar{V} ; 1,2)+f_{\bar{V}}(\bar{V} ; 2,2)\right. \\
& \left.-(1+\bar{V}) \exp (-\bar{V}) f_{\bar{V}}(\bar{V} ; 1,1)\right] \\
& -2 K_{2}\left[f_{\bar{V}}(\bar{V} ; 0,2)+\xi_{\bar{V}}(\bar{V} ; 1,2)\right. \\
& \left.-(1+\bar{V}) \exp (-\bar{V}) \xi_{\bar{V}}(\bar{V} ; 1,1)\right] .
\end{aligned}
$$

Finally, the zeroth-order solutions with zero AOA are derived, which are the fundamental for deriving the firstorder correction.

3.2.2. First-Order Correction. In this subsection, let us focus on the first-order dynamics. Regarding the dimensionless velocity, $\bar{V}$, as the dependent variable, the first-order dynamics can be formulated as

$$
\left\{\begin{array}{l}
\frac{d V_{b}^{(1)}}{d \bar{V}}=V_{e}\left[\left(K_{P}-1\right)-K_{a} \bar{C}_{d 0} \bar{V}^{2} \exp \left(-\bar{\beta} \Delta \bar{h}_{b}^{(0)}\right)-K_{2}\left(\sin \gamma_{0}+c_{1} \Delta \theta_{b}^{(0)}\right) \exp (-\bar{V})\right], \\
\frac{d \theta_{b}^{(1)}}{d \bar{V}}=-2\left(K_{1} \bar{V}-\frac{2 K_{2}}{\bar{V}}\right) \exp (-\bar{V}) \Delta \bar{h}_{b}^{(0)}+2\left(K_{1}+\frac{K_{2}}{\bar{V}^{2}}\right) \exp (-\bar{V}) \bar{V}_{b}^{(1)}, \\
\frac{d h_{b}^{(1)}}{d \bar{V}}=K_{1} r_{0} \exp (-\bar{V})\left[\bar{V}\left(\sin \gamma_{b}^{(0)}-c_{1} \Delta \theta_{b}^{(0)}-\sin \gamma_{0}\right)+\bar{V}_{b}^{(1)} \sin \gamma_{b}^{(0)}+\frac{\bar{V} \cos ^{2} \gamma_{b}^{(0)} \theta_{b}^{(1)}}{2}\right],
\end{array}\right.
$$




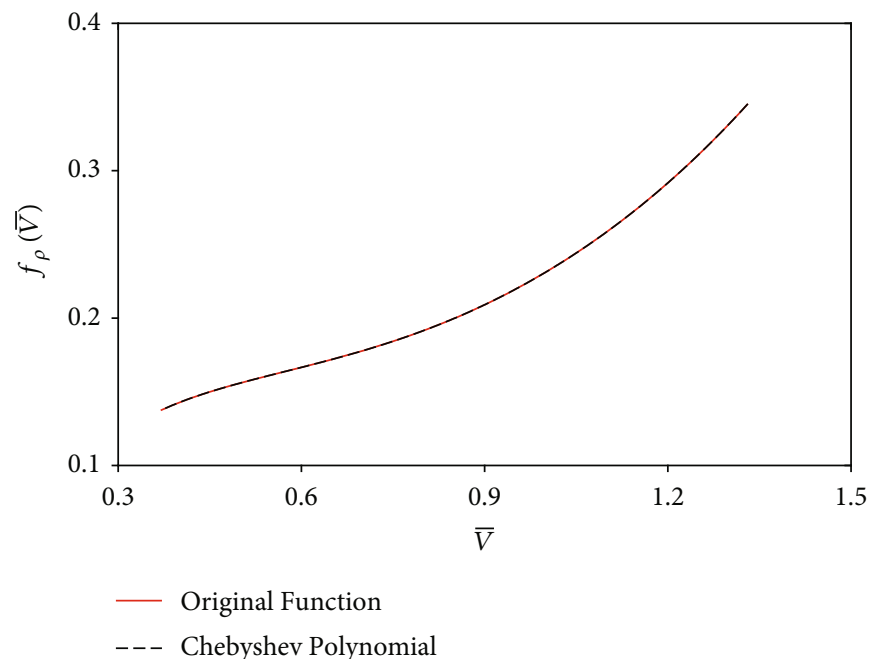

FIGURE 2: Comparison between $f_{\rho}(\bar{V})$ and the corresponding approximate polynomial.

where $\Delta \bar{h}_{b}^{(0)}=\left(h_{b}^{(0)}-h_{0}\right) / r_{0}$ and $\bar{V}_{b}^{(1)}=V_{b}^{(1)} / V_{e}$ are the dimensionless variables and $\bar{\beta}=\beta r_{0}, K_{P}=\bar{P} / F_{\text {ave }}$, and $K_{a}=$ $\rho_{i} S V_{e}^{2} / 2 F_{\text {ave }}$ are the constant coefficients.

The analytical solution to $V_{b}^{(1)}$ can be derived by integrating the first equation of (38). But the term, which is the integration of $\bar{V}^{2} \exp \left(-\bar{\beta} \Delta \bar{h}_{b}^{(0)}\right)$, cannot be solved analytically because $\Delta \bar{h}_{b}^{(0)}$ is not the linear function of $\bar{V}$. Similar to $(30)$, let

$$
f_{\rho}\left(x_{\bar{V}}\right)=x_{\bar{V}}^{2} \exp \left(-\bar{\beta} \Delta \bar{h}_{b}^{(0)}\right) \approx \sum_{i=0}^{N} f_{\rho}\left(x_{\bar{V} i}\right) L_{i}(x)
$$

where $\quad f_{\rho}\left(x_{\bar{V} i}\right)=x_{\bar{V}_{i}}^{2} \exp \left\{-\bar{\beta} K_{1}\left[\sin \gamma_{0} f_{\bar{V}}\left(x_{\bar{V} i} ; 1,1\right)+c_{1} I_{\theta}\right.\right.$ $\left.\left.\left(x_{\bar{V} i} ; 1,1\right)\right]\right\}$. As shown in Figure 2, the approximation polynomials are accurate enough in comparison with the original functions $f_{\rho}\left(x_{\bar{V}}\right)$. Then, the analytical solution to $V_{b}^{(1)}$ is

$$
\begin{aligned}
V_{b}^{(1)}(\bar{V})= & V_{e}\left\{\left(K_{P}-1\right)\left(\bar{V}-\bar{V}_{0}\right)\right. \\
& -\frac{1}{2}\left(\bar{V}-\bar{V}_{0}\right) K_{a} \bar{C}_{d 0} \sum_{i=0}^{N} f_{\rho}\left(x_{\bar{V} i}\right) W_{i}(x) \\
& -K_{2} \sin \gamma_{0}\left[\exp \left(-\bar{V}_{0}\right)-\exp (-\bar{V})\right] \\
& \left.-K_{2} c_{1} I_{\theta}(\bar{V} ; 0,1)\right\},
\end{aligned}
$$

where according to (33), the expression of $I_{\theta}(\bar{V} ; 0,1)$ is

$$
\begin{aligned}
I_{\theta}(\bar{V} ; 0,1)= & 2 K_{1}\left[f_{\bar{V}}(\bar{V} ; 1,2)-\exp (-\bar{V}) f_{\bar{V}}(\bar{V} ; 1,1)\right] \\
& -2 K_{2}\left[\xi_{\bar{V}}(\bar{V} ; 1,2)-\exp (-\bar{V}) \xi_{\bar{V}}(\bar{V} ; 1,1)\right]
\end{aligned}
$$

By integrating the second equation of (38), the analytical solution to $\theta_{b}^{(1)}$ can be obtained as

$$
\begin{aligned}
\theta_{b}^{(1)}(\bar{V})= & -2 K_{1}\left[\bar{h}_{r 0}^{(0)}+\bar{h}_{r 1}^{(0)}-\Delta \bar{h}_{b}^{(0)}(1+\bar{V}) \exp (-\bar{V})\right] \\
& +2 K_{1}\left[\bar{V}_{r}^{(1)}-\bar{V}_{b}^{(1)} \exp (-\bar{V})\right] \\
& +4 K_{2} \int_{V_{0}}^{\bar{V}} \frac{\Delta \bar{h}_{b}^{(0)} \exp \left(-x_{\bar{V})}\right)}{x_{\bar{V}}} d x_{\bar{V}} \\
& +2 K_{2} \int_{V_{0}}^{\bar{V}} \frac{\bar{V}_{b}^{(1)} \exp \left(-x_{\bar{V}}\right)}{x_{\bar{V}}^{2}} d x_{\bar{V}},
\end{aligned}
$$

where $\bar{h}_{r 0}^{(0)}, \bar{h}_{r 1}^{(0)}$, and $\bar{V}_{r}^{(1)}$ are all the functions of $\bar{V}$, which are expressed as

$$
\begin{aligned}
\bar{h}_{r 0}^{(0)}(\bar{V})= & K_{1}\left[\sin \gamma_{0} f_{\bar{V}}(\bar{V} ; 1,2)+c_{1} I_{\theta}(\bar{V} ; 1,2)\right], \\
\bar{h}_{r 1}^{(0)}(\bar{V})= & K_{1}\left[\sin \gamma_{0} f_{\bar{V}}(\bar{V} ; 2,2)+c_{1} I_{\theta}(\bar{V} ; 2,2)\right], \\
\bar{V}_{r}^{(1)}(\bar{V})= & \left(K_{P}-1\right) f_{\bar{V}}(\bar{V} ; 0,1)-\frac{1}{2}\left(\bar{V}-\bar{V}_{0}\right) K_{a} \bar{C}_{d 0} \\
& \cdot \sum_{i=0}^{N} f_{\rho}\left(x_{\bar{V} i}\right) \exp \left(-x_{\bar{V} i}\right) W_{i}(x) \\
& -K_{2} \sin \gamma_{0} f_{\bar{V}}(\bar{V} ; 0,2)-K_{2} c_{1} I_{\theta}(\bar{V} ; 0,2),
\end{aligned}
$$

where the expression of $I_{\theta}(\bar{V} ; 0,2), I_{\theta}(\bar{V} ; 1,2)$, and $I_{\theta}(\bar{V}$; $1,2)$ can be obtained from (33).

As for the two integration terms in (42), there are no analytical solutions. Chebyshev interpolation polynomials 
are also adopted to approximate the integrands. Let

$$
\frac{\exp \left(-m x_{\bar{V}}\right)}{x_{\bar{V}}^{n}}=\sum_{i=0}^{N} c_{\bar{V} i}^{(n, m)} x_{\bar{V}}^{i},
$$

where the coefficient $c_{\bar{V} i}^{(n, m)}$ is

$$
c_{\bar{V} i}^{(n, m)}=\sum_{k=i}^{N} p_{\bar{V} k}^{(n, m)} C_{n}^{k} \frac{\left(-\bar{V}_{2} / \bar{V}_{1}\right)^{n-k}}{\bar{V}_{1}^{k}} .
$$

Then, the analytical solutions to the two integration terms are

$$
\begin{gathered}
\int_{V_{0}}^{\bar{V}} \Delta \bar{h}_{b}^{(0)} \frac{\exp \left(-x_{\bar{V}}\right)}{x_{\bar{V}}} d x_{\bar{V}}=\sum_{i=0}^{N} c_{\bar{V} i}^{(1,1)} I_{h}(\bar{V} ; i), \\
\int_{V_{0}}^{\bar{V}} \bar{V}_{b}^{(1)} \frac{\exp \left(-x_{\bar{V}}\right)}{x_{\bar{V}}^{2}} d x_{\bar{V}}=\sum_{i=0}^{N} c_{\bar{V} i}^{(2,1)} I_{\bar{V}}(\bar{V} ; i),
\end{gathered}
$$

where

$$
\begin{aligned}
& I_{h}(\bar{V} ; n)=\int_{\bar{V}_{0}}^{\bar{V}} \Delta \bar{h}_{b}^{(0)} x_{\bar{V}}^{n} \exp \left(-x_{\bar{V}}\right) d x_{\bar{V}}, \\
& I_{\bar{V}}(\bar{V} ; n)=\int_{\bar{V}_{0}}^{\bar{V}} \bar{V}_{b}^{(1)} x_{\bar{V}}^{n} \exp \left(-x_{\bar{V}}\right) d x_{\bar{V}} .
\end{aligned}
$$

By the formula of partial integration, the analytical solution to $I_{h}(\bar{V} ; n)$ and $I_{\bar{V}}(\bar{V} ; n)$ can be derived as

$$
\begin{aligned}
& I_{h}(\bar{V} ; n)=-n ! \sum_{k=0}^{n} \frac{1}{k !}\left[\Delta \bar{h}_{b}^{(0)} \bar{V}^{k} \exp (-\bar{V})-\vartheta_{h}(\bar{V} ; k)\right], \\
& I_{\bar{V}}(\bar{V} ; n)=-n ! \sum_{k=0}^{n} \frac{1}{k !}\left[\bar{V}_{b}^{(1)} \bar{V}^{k} \exp (-\bar{V})-\vartheta_{\bar{V}}(\bar{V} ; k)\right],
\end{aligned}
$$

where the expressions of $\vartheta_{h}(\bar{V} ; n)$ and $\vartheta_{\bar{V}}(\bar{V} ; n)$ are

$$
\begin{aligned}
\vartheta_{h}(\bar{V} ; n)= & K_{1}\left[\sin \gamma_{0} f_{\bar{V}}(\bar{V} ; n+1,2)+c_{1} I_{\theta}(\bar{V} ; n+1,2)\right], \\
\vartheta_{\bar{V}}(\bar{V} ; n)= & \left(K_{P}-1\right) f_{\bar{V}}(\bar{V} ; n, 1)-\frac{1}{2}\left(\bar{V}-\bar{V}_{0}\right) K_{a} C_{d 0} \\
& \cdot \sum_{i=0}^{N} f_{\rho}\left(x_{\bar{V} i}\right) x_{\bar{V} i}^{n} \exp \left(-x_{\bar{V} i}\right) W_{i}(\bar{V}) \\
& -K_{2} \sin \gamma_{0} f_{\bar{V}}(\bar{V} ; n, 2)-K_{2} c_{1} I_{\theta}(\bar{V} ; n, 2) .
\end{aligned}
$$

Then, the analytical solution to $\theta_{b}^{(1)}$ is

$$
\begin{aligned}
\theta_{b}^{(1)}(\bar{V})= & -2 K_{1}\left[\bar{h}_{r 0}^{(0)}+\bar{h}_{r 1}^{(0)}-\Delta \bar{h}_{b}^{(0)}(1+\bar{V}) \exp (-\bar{V})\right] \\
& +4 K_{2} \sum_{i=0}^{N} c_{\bar{V} i}^{(1,1)} I_{h}(\bar{V} ; i) \\
& +2 K_{1}\left[\bar{V}_{r}^{(1)}-\bar{V}_{b}^{(1)} \exp (-\bar{V})\right] \\
& +2 K_{2} \sum_{i=0}^{N} c_{\bar{V} i}^{(2,1)} I_{\bar{V}}(\bar{V} ; i)
\end{aligned}
$$

As for the analytical solution to $h_{b}^{(1)}$, it is the integral of $d h_{b}^{(0)} / d \bar{V}$ from $\bar{V}_{0}$ to $\bar{V}$. It is noted that all the variables in $d h_{b}^{(0)} / d \bar{V}$, such as $\gamma_{b}^{(0)}, \theta_{b}^{(0)}, \bar{V}_{b}^{(1)}$, and $\theta_{b}^{(1)}$, are expressed as the function of $\bar{V}$. Therefore, we can use Chebyshev interpolation polynomial to approximate the integrand. Let

$$
\begin{aligned}
f_{h}\left(x_{\bar{V}}\right)= & \exp \left(-x_{\bar{V}}\right)\left[x_{\bar{V}}\left(\sin \gamma_{b}^{(0)}-c_{1} \Delta \theta_{b}^{(0)}-\sin \gamma_{0}\right)\right. \\
& \left.+\bar{V}_{b}^{(1)} \sin \gamma_{b}^{(0)}+\frac{x_{\bar{V}} \cos ^{2} \gamma_{b}^{(0)} \theta_{b}^{(1)}}{2}\right] \\
\approx & \sum_{i=0}^{N} f_{h}\left(x_{\bar{V} i}\right) L_{i}(x)
\end{aligned}
$$

Then, the solution to $h_{b}^{(1)}$ can be obtained as

$$
h_{b}^{(1)}(\bar{V})=K_{1} r_{0} \sum_{i=0}^{N} f_{h}\left(x_{\bar{V} i}\right) W_{i}(\bar{V}) \text {. }
$$

Finally, the first-order solutions are derived. According to (A.6), the analytical solution with zero AOA is the sum of the zero-order and first-order solutions.

3.3. Analytic Ascent Trajectory with Arbitrary AOA Profile. In the last subsection, the analytical solutions to ascent trajectory with zero AOA are provided. In order to obtain the full analytical solutions to ascent trajectory with arbitrary AOA profile, the analytical solutions to trajectory increment corresponding to no-zero AOA are presented firstly in this subsection. Then, the full analytical solutions are the sum of analytical trajectory with zero AOA and trajectory increment corresponding to nonzero AOA.

3.3.1. Regular Perturbation Model with AOA Increment. Because the independent variable for the analytical solution with zero $\mathrm{AOA}$ is the dimensionless velocity, $\bar{V}$, the 
differential equations of trajectory increments should be formulated as

$$
\left\{\begin{array}{l}
\frac{d \Delta V}{d \bar{V}}=V_{e}\left(f_{V 1} \alpha^{2}+f_{V 2} \frac{\Delta V}{V_{e}}+f_{V 3} \Delta \gamma+f_{V 4} \frac{\Delta h}{r_{0}}+f_{V 5} \frac{\Delta h \alpha^{2}}{r_{0}}+f_{V 6} \Delta \gamma^{2}\right), \\
\frac{d \Delta \gamma}{d \bar{V}}=f_{\gamma 1} \alpha+f_{\gamma 2} \frac{\Delta V}{V_{e}}+f_{\gamma 3} \Delta \gamma+f_{\gamma 4} \frac{\Delta h \alpha}{r_{0}}+f_{\gamma 5} \Delta \gamma^{2}, \\
\frac{d \Delta \bar{h}}{d \bar{V}}=r_{0}\left(f_{h 1} \frac{\Delta V}{V_{e}}+f_{h 2} \Delta \gamma+f_{h 3} \frac{\Delta V \Delta \gamma}{V_{e}}+f_{h 4} \Delta \gamma^{2}\right),
\end{array},\right.
$$

where $f_{V 1} \sim f_{V 6}, f_{\gamma 1} \sim f_{\gamma 5}$, and $f_{h 1} \sim f_{h 4}$ are all the functions of $\bar{V}$, which are expressed as

$$
\begin{aligned}
& f_{V 1}(\bar{V})=-\frac{K_{P}}{2}+K_{a} \bar{C}_{d}^{\alpha} \bar{V}_{b}^{2} \exp \left(-\beta \Delta h_{b}\right), \\
& f_{V 2}(\bar{V})=-2 K_{a} \bar{C}_{d 0} \bar{V}_{b} \exp \left(-\beta \Delta h_{b}\right), \\
& f_{V 3}(\bar{V})=-K_{2} \cos \gamma_{b} \exp (-\bar{V}), \\
& f_{V 4}(\bar{V})=\beta r_{0} K_{a} C_{d 0} \bar{V}_{b}^{2} \exp \left(-\beta \Delta h_{b}\right), \\
& f_{V 5}(\bar{V})=\beta r_{0} K_{a} \bar{C}_{d}^{\alpha} \bar{V}_{b}^{2} \exp \left(-\beta \Delta h_{b}\right), \\
& f_{V 6}(\bar{V})=\frac{K_{2} \sin \gamma_{b} \exp (-\bar{V})}{2}, \\
& f_{\gamma 1}(\bar{V})=\frac{K_{P}}{\bar{V}_{b}}+K_{a} \bar{C}_{l}^{\alpha} \bar{V}_{b} \exp \left(-\beta \Delta h_{b}\right), \\
& f_{\gamma 2}(\bar{V})=\left(K_{1}+\frac{K_{2}}{\bar{V}_{b}^{2}}\right) \cos \gamma_{b} \exp (-\bar{V}), \\
& f_{\gamma 3}(\bar{V})=-\left(K_{1} \bar{V}_{b}-\frac{K_{2}}{\bar{V}_{b}}\right) \sin \gamma_{b} \exp (-\bar{V}), \\
& f_{\gamma 4}(\bar{V})=-\beta r_{0} K_{a} \bar{C}_{l}^{\alpha} \bar{V}_{b} \exp \left(-\beta \Delta h_{b}\right), \\
& f_{\gamma 5}(\bar{V})=-\frac{\left(K_{1} \bar{V}_{b}-K_{2} / \bar{V}_{b}\right) \cos \gamma_{b} \exp (-\bar{V})}{2},
\end{aligned}
$$

$$
\begin{aligned}
& f_{h 1}(\bar{V})=K_{1} \sin \gamma_{b} \exp (-\bar{V}), \\
& f_{h 2}(\bar{V})=K_{1} \bar{V}_{b} \cos \gamma_{b} \exp (-\bar{V}), \\
& f_{h 3}(\bar{V})=K_{1} \cos \gamma_{b} \exp (-\bar{V}), \\
& f_{h 4}(\bar{V})=-\frac{K_{1} \bar{V}_{b} \sin \gamma_{b} \exp (-\bar{V})}{2} .
\end{aligned}
$$

Generally, the AOA profile is expressed as the function of the flight time. The AOA profile with the dimensionless velocity can be formulated by using the relationship between the flight time $t$ and $\bar{V}$, which can be easily expressed as

$$
t=t_{0}+T\left[1-\exp \left(\bar{V}_{0}-\bar{V}\right)\right]
$$

In the differential equation of $\Delta \gamma$, it is easy to find that the term, $f_{\gamma 1} \alpha$, is much larger than the sum of other terms, $f_{\gamma 1} \alpha$ $\gg f_{\gamma 2} \Delta V / V_{e}+f_{\gamma 3} \Delta \gamma+f_{\gamma 4} \Delta h \alpha / r_{0}+f_{\gamma 5} \Delta \gamma^{2}$, which is the dominant term. Similarly, the term, $f_{V 1} \alpha^{2}+f_{V 3} \Delta \gamma$, is the dominant term of the differential equation of $\Delta V$. As for the differential equation of $\Delta h$, due to $V_{b} \gg \Delta V$ and $\Delta \gamma^{2} \leq 1$, it is obvious that the term, $f_{h 1} \Delta V / V_{e}+f_{h 2}$ $\Delta \gamma$, is the dominant term. Therefore, the dynamics can be modified as

$$
\left[\begin{array}{c}
\frac{d \Delta \gamma}{d \bar{V}} \\
\frac{d \Delta V}{d \bar{V}} \\
\frac{d \Delta h}{d \bar{V}}
\end{array}\right]=\left[\begin{array}{c}
f_{\gamma 1} \alpha \\
V_{e} f_{V 1} \alpha^{2}+V_{e} f_{V 3} \Delta \gamma \\
\frac{r_{0}}{V_{e}} f_{h 1} \Delta V+r_{0} f_{h 2} \Delta \gamma
\end{array}\right]+\frac{\varepsilon}{k}\left[\begin{array}{c}
\frac{1}{V_{e}} f_{\gamma 2} \Delta V+f_{\gamma 3} \Delta \gamma+\frac{1}{r_{0}} f_{\gamma 4} \Delta h \alpha+f_{\gamma 5} \Delta \gamma^{2} \\
f_{V 2} \Delta V+\frac{V_{e}}{r_{0}} f_{V 4} \Delta h+\frac{V_{e}}{r_{0}} f_{V 5} \Delta h \alpha^{2}+V_{e} f_{V 6} \Delta \gamma^{2} \\
\frac{r_{0}}{V_{e}} f_{h 3} \Delta V \Delta \gamma+r_{0} f_{h 4} \Delta \gamma^{2}
\end{array}\right]
$$


According to the regular perturbation method presented in Appendix B, the zeroth-order and first-order dynamics are

$$
\begin{aligned}
& \left\{\begin{array}{l}
\frac{d \Delta \gamma^{(0)}}{d \bar{V}}=f_{\gamma 1}(\bar{V}) \alpha, \\
\frac{d \Delta V^{(0)}}{d \bar{V}}=V_{e}\left[f_{V 1}(\bar{V}) \alpha^{2}+f_{V 3}(\bar{V}) \Delta \gamma^{(0)}\right], \\
\frac{d \Delta h^{(0)}}{d \bar{V}}=r_{0}\left[f_{h 1}(\bar{V}) \frac{\Delta V^{(0)}}{V_{e}}+f_{h 2}(\bar{V}) \Delta \gamma^{(0)}\right],
\end{array}\right. \\
& \left\{\begin{array}{l}
\frac{d \Delta \gamma^{(1)}}{d \bar{V}}=f_{\gamma 2}(\bar{V}) \frac{\Delta V^{(0)}}{V_{e}}+f_{\gamma 3}(\bar{V}) \Delta \gamma^{(0)}+f_{\gamma 4}(\bar{V}) \frac{\Delta h^{(0)} \alpha}{r_{0}}+f_{\gamma 5}(\bar{V})\left(\Delta \gamma^{(0)}\right)^{2}, \\
\frac{d \Delta V^{(1)}}{d \bar{V}}=V_{e}\left[f_{V 2}(\bar{V}) \frac{\Delta V^{(0)}}{V_{e}}+f_{V 4}(\bar{V}) \frac{\Delta h^{(0)}}{r_{0}}+f_{V 5}(\bar{V}) \frac{\Delta h^{(0)} \alpha^{2}}{r_{0}}+f_{V 6}(\bar{V})\left(\Delta \gamma^{(0)}\right)^{2}\right], \\
\frac{d \Delta h^{(1)}}{d \bar{V}}=r_{0}\left[f_{h 1}(\bar{V}) \frac{\Delta V^{(1)}}{V_{e}}+f_{h 2}(\bar{V}) \Delta \gamma^{(1)}+f_{h 3}(\bar{V}) \frac{\Delta V^{(0)} \Delta \gamma^{(0)}}{V_{e}}+f_{h 3}(\bar{V})\left(\Delta \gamma^{(0)}\right)^{2}\right] .
\end{array}\right.
\end{aligned}
$$

The initial values of zeroth-order and first-order states are all zeros.

3.3.2. Zeroth-Order Solution to Trajectory Increment. It is obvious that the three differential equations of zero-order states are not coupled. Therefore, by integrating these differential equations orderly, the analytical solutions to $\Delta \gamma^{(0)}$, $\Delta V^{(0)}$, and $\Delta h^{(0)}$ can be obtained as

$$
\begin{gathered}
\Delta \gamma^{(0)}(\bar{V})=\int_{\bar{V}_{0}}^{\bar{V}} f_{\gamma 1}\left(x_{\bar{V}}\right) \alpha\left(x_{\bar{V}}\right) d x_{\bar{V}}, \\
\Delta V^{(0)}(\bar{V})=V_{e} \int_{\bar{V}_{0}}^{\bar{V}} f_{V 1}\left(x_{\bar{V}}\right) \alpha^{2}\left(x_{\bar{V}}\right) d x_{\bar{V}} \\
+V_{e} \int_{\bar{V}_{0}}^{\bar{V}} f_{V 3}\left(x_{\bar{V}}\right) \Delta \gamma^{(0)}\left(x_{\bar{V}}\right) d x_{\bar{V}}, \\
\Delta h^{(0)}(\bar{V})= \\
\quad \frac{r_{0}}{V_{e}} \int_{\bar{V}_{0}}^{\bar{V}} f_{h 1}\left(x_{\bar{V}}\right) \Delta V^{(0)}\left(x_{\bar{V}}\right) d x_{\bar{V}} \\
+r_{0} \int_{\bar{V}_{0}}^{\bar{V}} f_{h 2}\left(x_{\bar{V}}\right) \Delta \gamma^{(0)}\left(x_{\bar{V}}\right) d x_{\bar{V}} .
\end{gathered}
$$

Although the integral terms in the above formulas are quite complex, the integrands are related only to the independent variable, $\bar{V}$. Here, Chebyshev polynomials are also used to approximate the integrands. Let

$$
f_{\gamma 1}\left(x_{\bar{V}}\right) \alpha\left(x_{\bar{V}}\right) \approx \sum_{i=0}^{N} f_{\gamma 1}\left(x_{\bar{V} i}\right) \alpha\left(x_{\bar{V} i}\right) L_{i}(x)
$$

$$
\begin{gathered}
f_{V 1}\left(x_{\bar{V}}\right) \alpha^{2}\left(x_{\bar{V}}\right) \approx \sum_{i=0}^{N} f_{V 1}\left(x_{\bar{V} i}\right) \alpha^{2}\left(x_{\bar{V} i}\right) L_{i}(x), \\
f_{V 3}\left(x_{\bar{V}}\right) \Delta \gamma^{(0)}\left(x_{\bar{V}}\right) \approx \sum_{i=0}^{N} f_{V 3}\left(x_{\bar{V} i}\right) \Delta \gamma^{(0)}\left(x_{\bar{V} i}\right) L_{i}(x), \\
f_{h 1}\left(x_{\bar{V}}\right) \Delta \bar{V}^{(0)}\left(x_{\bar{V}}\right) \approx \sum_{i=0}^{N} f_{h 1}\left(x_{\bar{V} i}\right) \Delta \bar{V}^{(0)}\left(x_{\bar{V} i}\right) L_{i}(x), \\
f_{h 2}\left(x_{\bar{V}}\right) \Delta \gamma^{(0)}\left(x_{\bar{V}}\right) \approx \sum_{i=0}^{N} f_{h 2}\left(x_{\bar{V} i}\right) \Delta \gamma^{(0)}\left(x_{\bar{V} i}\right) L_{i}(x) .
\end{gathered}
$$

Substituting (62) into (59), the analytical solution to the zeroth-order flight-path angle increment can be obtained as

$$
\Delta \gamma^{(0)}(\bar{V})=\frac{1}{2}\left(\bar{V}-\bar{V}_{0}\right) \sum_{i=0}^{N} f_{\gamma 1}\left(x_{\bar{V} i}\right) \alpha\left(x_{\bar{V} i}\right) W_{i}(x)=\gamma_{\alpha 1}^{(0)}(\bar{V}) \alpha,
$$

where $\alpha$ is the vector consisting of the value of AOA at interpolating points, i.e.,

$$
\alpha=\left[\alpha\left(x_{\bar{V} 0}\right), \alpha\left(x_{\bar{V} 1}\right), \cdots, \alpha\left(x_{\bar{V} N}\right)\right]^{\mathrm{T}},
$$

$\gamma_{\alpha 1}^{(0)}(\bar{V})$ is the coefficient matrix expressed as

$$
\begin{aligned}
\gamma_{\alpha 1}^{(0)}(\bar{V}) & =\frac{1}{2}\left(\bar{V}-\bar{V}_{0}\right)\left[W_{0}(\bar{V}) f_{\gamma 1}\left(x_{\bar{V} 0}\right), \cdots, W_{N}(\bar{V}) f_{\gamma 1}\left(x_{\bar{V} N}\right)\right] \\
& =W^{T}(\bar{V}) F_{\gamma 1},
\end{aligned}
$$


where

$$
W(\bar{V})=\frac{1}{2}\left(\bar{V}-\bar{V}_{0}\right)\left[W_{0}(x), \cdots, W_{N}(x)\right]^{\mathrm{T}} .
$$

$F_{\gamma 1}$ is the diagonal matrix whose diagonal elements are the value of $f_{\gamma 1}$ at interpolating points, i.e.,

$$
F_{\gamma 1}=\left[\begin{array}{ccc}
f_{\gamma 1}\left(x_{\bar{V} 0}\right) & \cdots & 0 \\
\vdots & \ddots & \vdots \\
0 & \cdots & f_{\gamma 1}\left(x_{\bar{V} N}\right)
\end{array}\right]
$$

Similarly, $F_{\gamma 2} \sim F_{\gamma 3}, F_{V 1} \sim F_{V 4}$, and $F_{h 1} \sim F_{h 2}$ mentioned below are all the diagonal matrices whose diagonal elements are the value of corresponding function at interpolating points.

Substituting (63) and (64) into (60), the analytical solution to the zeroth-order velocity increment can be obtained as

$$
\begin{aligned}
\Delta V^{(0)}(\bar{V})= & \frac{1}{2}\left(\bar{V}-\bar{V}_{0}\right) V_{e} \sum_{i=0}^{N}\left[f_{V 1}\left(x_{\bar{V} i}\right) \alpha^{2}\left(x_{\bar{V} i}\right)\right. \\
& \left.+f_{V 3}\left(x_{\bar{V} i}\right) \Delta \gamma^{(0)}\left(x_{\bar{V} i}\right)\right] W_{i}(x) \\
= & \frac{1}{2}\left(\bar{V}-\bar{V}_{0}\right) V_{e} \sum_{i=0}^{N}\left[f_{V 1}\left(x_{\bar{V} i}\right) \alpha^{2}\left(x_{\bar{V} i}\right)\right. \\
& \left.+f_{V 3}\left(x_{\bar{V} i}\right) \gamma_{\alpha 1}^{(0)}\left(x_{\bar{V} i}\right) \alpha\right] W_{i}(x) \\
= & V_{\alpha 1}^{(0)}(\bar{V}) \alpha+V_{\alpha 2}^{(0)}(\bar{V}) \alpha_{\text {squ }},
\end{aligned}
$$

where $\alpha_{\text {squ }}$ is the vector consisting of the square of $\mathrm{AOA}$ at interpolating points, i.e.,

$$
\alpha_{\text {squ }}=\left[\alpha^{2}\left(x_{\bar{V} 0}\right), \alpha^{2}\left(x_{\bar{V} 1}\right), \cdots, \alpha^{2}\left(x_{\bar{V} N}\right)\right]^{\mathrm{T}},
$$

$V_{\alpha 1}^{(0)}(\bar{V})$ and $V_{\alpha 2}^{(0)}(\bar{V})$ are the coefficient matrices expressed as

$$
V_{\alpha 1}^{(0)}(\bar{V})=V_{e} W^{\mathrm{T}}(\bar{V}) F_{V 3} A_{c b} F_{\gamma 1}, V_{\alpha 2}^{(0)}(\bar{V})=V_{e} W^{\mathrm{T}}(\bar{V}) F_{V 1},
$$

where $A_{c b}$ is

$$
A_{c b}=\left[W\left(x_{\bar{V} 0}\right), \cdots, W\left(x_{\bar{V} N}\right)\right]^{\mathrm{T}} .
$$

Substituting (65) and (66) into (61), the analytical solution to the zeroth-order altitude increment can be obtained as

$$
\begin{aligned}
\Delta h^{(0)}(\bar{V})= & \frac{1}{2}\left(\bar{V}-\bar{V}_{0}\right) r_{0} \sum_{i=0}^{N}\left[f_{h 1}\left(x_{\bar{V} i}\right) \Delta \bar{V}^{(0)}\left(x_{\bar{V} i}\right)\right. \\
& \left.+f_{h 2}\left(x_{\bar{V} i}\right) \Delta \gamma^{(0)}\left(x_{\bar{V} i}\right)\right] W_{i}(x) \\
= & \frac{1}{2}\left(\bar{V}-\bar{V}_{0}\right) r_{0} \sum_{i=0}^{N} f_{h 1}\left(x_{\bar{V} i}\right) \\
& \cdot \frac{V_{\alpha 1}^{(0)}(\bar{V}) \alpha+V_{\alpha 2}^{(0)}(\bar{V}) \alpha_{\text {squ }}}{V_{e}} W_{i}(x) \\
& +\frac{1}{2}\left(\bar{V}-\bar{V}_{0}\right) r_{0} \sum_{i=0}^{N} f_{h 2}\left(x_{\bar{V} i}\right) W^{T}(\bar{V}) F_{\gamma 1} \alpha W_{i}(x) \\
= & h_{\alpha 1}^{(0)}(\bar{V}) \alpha+h_{\alpha 2}^{(0)}(\bar{V}) \alpha_{\mathrm{squ}},
\end{aligned}
$$

where

$$
\begin{aligned}
& h_{\alpha 1}^{(0)}(\bar{V})=r_{0} W^{T}(\bar{V})\left(F_{h 1} A_{c b} F_{V 3}+F_{h 2}\right) A_{c b} F_{\gamma 1}, \\
& h_{\alpha 2}^{(0)}(\bar{V})=r_{0} W(\bar{V}) F_{h 1} A_{c b} F_{V 1} .
\end{aligned}
$$

3.3.3. First-Order Correction to Trajectory Increment. As for the first-order corrections, their analytical solutions can be obtained by integrating the three differential equations of (58) in sequence. Similar to the zeroth-order solution, Chebyshev polynomials are also used to approximate the integrands. Therefore, the analytical solution to $\Delta \gamma^{(1)}$ is

$$
\begin{aligned}
\Delta \gamma^{(1)}(\bar{V})= & \frac{1}{2}\left(\bar{V}-\bar{V}_{0}\right) \sum_{i=0}^{N} W_{i}(x) \\
& \cdot\left[f_{\gamma 2}\left(x_{\bar{V} i}\right) \frac{\Delta V^{(0)}\left(x_{\bar{V} i}\right)}{V_{e}}+f_{\gamma 3}\left(x_{\bar{V} i}\right) \Delta \gamma^{(0)}\left(x_{\bar{V} i}\right)\right] \\
& +\frac{1}{2}\left(\bar{V}-\bar{V}_{0}\right) \sum_{i=0}^{N} W_{i}(x) \\
& \cdot\left[f_{\gamma 4}\left(x_{\bar{V} i}\right) \frac{\Delta h^{(0)}\left(x_{\bar{V} i}\right) \alpha\left(x_{\bar{V} i}\right)}{r_{0}} f_{\gamma 5}\left(x_{\bar{V} i}\right)\left(\Delta \gamma^{(0)}\left(x_{\bar{V} i}\right)\right)^{2}\right] .
\end{aligned}
$$

Similar to (76), the first term of (78) can be rewritten as

$$
\Delta \gamma_{1}^{(1)}(\bar{V})=\gamma_{\alpha 1}^{(1)}(\bar{V}) \boldsymbol{\alpha}+\gamma_{\alpha 2}(\bar{V}) \boldsymbol{\alpha}_{\text {squ }},
$$

where

$$
\begin{aligned}
\gamma_{\alpha 1}^{(1)}(\bar{V}) & =W^{\mathrm{T}}(\bar{V})\left(F_{\gamma 2} A_{c b} F_{V 3}+F_{\gamma 3}\right) A_{c b} F_{\gamma 1}, \\
\gamma_{\alpha 2}(\bar{V}) & =W^{\mathrm{T}}(\bar{V}) F_{\gamma 2} A_{c b} F_{V 1} .
\end{aligned}
$$


Substituting (69) and (76) into the second term of (78), it can be expressed as

$$
\begin{aligned}
\Delta \gamma_{2}^{(1)}(\bar{V})= & \frac{1}{2}\left(\bar{V}-\bar{V}_{0}\right) \frac{1}{r_{0}} \sum_{i=0}^{N} W_{i}(x) f_{\gamma 4}\left(x_{\bar{V} i}\right) \\
& \cdot\left(h_{\alpha 1}^{(0)}\left(x_{\bar{V} i}\right) \alpha+h_{\alpha 2}^{(0)}\left(x_{\bar{V} i}\right) \alpha_{\text {squ }}\right) \alpha\left(x_{\bar{V} i}\right) \\
& +\frac{1}{2}\left(\bar{V}-\bar{V}_{0}\right) \sum_{i=0}^{N} W_{i}(x) f_{\gamma 5}\left(x_{\bar{V} i}\right)\left(\gamma_{\alpha 1}^{(0)}\left(x_{\bar{V} i}\right) \alpha\right)^{2} \\
= & \alpha^{T} \gamma_{\alpha 3}(\bar{V}) \alpha+\alpha_{\text {squ }}^{\mathrm{T}} \gamma_{\alpha 4}(\bar{V}) \alpha
\end{aligned}
$$

where $\gamma_{\alpha 3}(\bar{V})$ and $\gamma_{\alpha 4}(\bar{V})$ are the coefficient matric expressed as

$$
\begin{aligned}
\gamma_{\alpha 3}(\bar{V})= & \frac{1}{2}\left(\bar{V}-\bar{V}_{0}\right) \frac{1}{r_{0}} \sum_{i=0}^{N} W_{i}(\bar{V}) f_{\gamma 4}\left(x_{\bar{V} i}\right)\left[h_{\alpha 1}^{(0)}\left(x_{\bar{V} i}\right)\right]^{T} I(i) \\
& +\frac{1}{2}\left(\bar{V}-\bar{V}_{0}\right) \sum_{i=0}^{N} W_{i}(\bar{V}) f_{\gamma 5}\left(x_{\bar{V} i}\right)\left[\gamma_{\alpha 1}^{(0)}\left(x_{\bar{V} i}\right)\right]^{T} \gamma_{\alpha 1}^{(0)}\left(x_{\bar{V} i}\right), \\
\gamma_{\alpha 4}(\bar{V})= & \frac{1}{2}\left(\bar{V}-\bar{V}_{0}\right) \frac{1}{r_{0}} \sum_{i=0}^{N} W_{i}(\bar{V}) f_{\gamma 4}\left(x_{\bar{V} i}\right)\left[h_{\alpha 2}^{(0)}\left(x_{\bar{V} i}\right)\right]^{T} I(i),
\end{aligned}
$$

where $I(i)$ is the $i$-th row of the identity matrix with the dimension $N+1$. Then, combining (79) with (81), we get

$$
\begin{aligned}
\Delta \gamma^{(1)}(\bar{V})= & \gamma_{\alpha 1}^{(1)}(\bar{V}) \alpha+\gamma_{\alpha 2}(\bar{V}) \alpha_{\text {squ }} \\
& +\alpha^{\mathrm{T}} \gamma_{\alpha 3}(\bar{V}) \alpha+\alpha_{\text {squ }}^{\mathrm{T}} \gamma_{\alpha 4}(\bar{V}) \alpha .
\end{aligned}
$$

By using Chebyshev polynomials to approximate the integrands, the analytical solution to $\Delta V^{(1)}$ can be obtained as

$$
\begin{aligned}
\Delta V^{(1)}(\bar{V}) & \\
= & \frac{1}{2}\left(\bar{V}-\bar{V}_{0}\right) V_{e} \sum_{i=0}^{N} W_{i}(x) \\
& \cdot\left[f_{V 2}\left(x_{\bar{V} i}\right) \frac{\Delta V^{(0)}\left(x_{\bar{V} i}\right)}{V_{e}}+f_{V 4}\left(x_{\bar{V} i}\right) \frac{\Delta h^{(0)}\left(x_{\bar{V} i}\right)}{r_{0}}\right] \\
+ & \frac{1}{2}\left(\bar{V}-\bar{V}_{0}\right) V_{e} \sum_{i=0}^{N} W_{i}(x) \\
& \cdot\left[f_{V 5}\left(x_{\bar{V} i}\right) \frac{\Delta h^{(0)}\left(x_{\bar{V} i}\right) \alpha^{2}\left(x_{\bar{V} i}\right)}{r_{0}}+f_{V 6}\left(x_{\bar{V} i}\right)\left(\Delta \gamma^{(0)}\left(x_{\bar{V} i}\right)\right)^{2}\right] .
\end{aligned}
$$

Similar to (76), the first term of (84) can be rewritten as

$$
\Delta V_{1}^{(1)}(\bar{V})=V_{\alpha 1}^{(0)}(\bar{V}) \alpha+V_{\alpha 2}^{(0)}(\bar{V}) \alpha_{\text {squ }}
$$

where

$$
\begin{aligned}
V_{\alpha 1}^{(1)}(\bar{V})= & V_{e} W^{\mathrm{T}}(\bar{V})\left[F_{V 2} A_{c b} F_{V 3}\right. \\
& \left.+F_{V 4} A_{c b}\left(F_{h 1} A_{c b} F_{V 3}+F_{h 2}\right)\right] A_{c b} F_{\gamma 1}, \\
V_{\alpha 2}^{(1)}(\bar{V})= & V_{e} W^{\mathrm{T}}(\bar{V})\left(F_{V 2}+F_{V 4} A_{c b} F_{h 1}\right) A_{c b} F_{V 1} .
\end{aligned}
$$

Similarly, substituting (69) and (76) into the second term of (84), it can be expressed as

$$
\begin{aligned}
\Delta V_{2}^{(1)}(\bar{V})= & \frac{1}{2}\left(\bar{V}-\bar{V}_{0}\right) \frac{V_{e}}{r_{0}} \sum_{i=0}^{N} W_{i}(x) f_{V 5}\left(x_{\bar{V} i}\right) \\
& \cdot\left(h_{\alpha 1}^{(0)}\left(x_{\bar{V} i}\right) \alpha+h_{\alpha 2}^{(0)}\left(x_{\bar{V} i}\right) \alpha_{\text {squ }}\right) \alpha^{2}\left(x_{\bar{V} i}\right) \\
& +\frac{1}{2}\left(\bar{V}-\bar{V}_{0}\right) V_{e} \sum_{i=0}^{N} W_{i}(x) f_{V 6}\left(x_{\bar{V} i}\right)\left(\gamma_{\alpha 1}^{(0)}\left(x_{\bar{V} i}\right) \alpha\right)^{2} \\
= & \alpha^{T} V_{\alpha 3}(\bar{V}) \alpha+\alpha_{\text {squ }}^{\mathrm{T}} V_{\alpha 4}(\bar{V}) \alpha+\alpha_{\text {squ }}^{\mathrm{T}} V_{\alpha 5}(\bar{V}) \alpha_{\text {squ }},
\end{aligned}
$$

where

$$
\begin{aligned}
& V_{\alpha 3}(\bar{V})=\frac{1}{2}\left(\bar{V}-\bar{V}_{0}\right) V_{e} \sum_{i=0}^{N} W_{i}(x) f_{V 6}\left(x_{\bar{V} i}\right)\left[\gamma_{\alpha 1}^{(0)}\left(x_{\bar{V} i}\right)\right]^{\mathrm{T}} \gamma_{\alpha 1}^{(0)}\left(x_{\bar{V} i}\right), \\
& V_{\alpha 4}(\bar{V})=\frac{1}{2}\left(\bar{V}-\bar{V}_{0}\right) \frac{V_{e}}{r_{0}} \sum_{i=0}^{N} W_{i}(x) f_{V 5}\left(x_{\bar{V} i}\right) I^{T}(i) h_{\alpha 1}^{(0)}\left(x_{\bar{V} i}\right), \\
& V_{\alpha 5}(\bar{V})=\frac{1}{2}\left(\bar{V}-\bar{V}_{0}\right) \frac{V_{e}}{r_{0}} \sum_{i=0}^{N} W_{i}(x) f_{V 5}\left(x_{\bar{V} i}\right) I^{T}(i) h_{\alpha 2}^{(0)}\left(x_{\bar{V} i}\right) .
\end{aligned}
$$

Then, combining (85) with (87), the analytical solution to $\Delta V^{(1)}$ is

$$
\begin{aligned}
\Delta V^{(1)}(\bar{V})= & V_{\alpha 1}^{(0)}(\bar{V}) \alpha+V_{\alpha 2}^{(0)}(\bar{V}) \alpha_{\text {squ }}+\alpha^{\mathrm{T}} V_{\alpha 3}(\bar{V}) \alpha \\
& +\alpha_{\text {squ }}^{\mathrm{T}} V_{\alpha 4}(\bar{V}) \alpha+\alpha_{\text {squ }}^{\mathrm{T}} V_{\alpha 5}(\bar{V}) \alpha_{\text {squ }} .
\end{aligned}
$$

Similar to (78) and (84), the analytical solution to $\Delta h^{(1)}$ can be approximated as

$$
\begin{aligned}
\Delta h^{(1)}(\bar{V})= & \frac{1}{2}\left(\bar{V}-\bar{V}_{0}\right) r_{0} \sum_{i=0}^{N} W_{i}(x) f_{h 1}\left(x_{\bar{V} i}\right) \frac{\Delta V^{(1)}\left(x_{\bar{V} i}\right)}{V_{e}} \\
& +r_{0} \sum_{i=0}^{N} W_{i}(\bar{V}) f_{h 2}\left(x_{\bar{V} i}\right) \Delta \gamma^{(1)}\left(x_{\bar{V} i}\right) \\
& +\frac{1}{2}\left(\bar{V}-\bar{V}_{0}\right) r_{0} \sum_{i=0}^{N} W_{i}(x) \\
& \cdot\left[f_{h 3}\left(x_{\bar{V} i}\right) \frac{\Delta V^{(0)}\left(x_{\bar{V} i}\right) \Delta \gamma^{(0)}\left(x_{\bar{V} i}\right)}{V_{e}}+f_{h 4}\left(x_{\bar{V} i}\right)\left(\Delta \gamma^{(0)}\left(x_{\bar{V} i}\right)\right)^{2}\right] .
\end{aligned}
$$


Substituting (89) into (90), the first term of (90) can be rewritten as

$$
\begin{aligned}
\Delta h_{1}^{(1)}(\bar{V})= & \frac{1}{2}\left(\bar{V}-\bar{V}_{0}\right) \frac{r_{0}}{V_{e}} \sum_{i=0}^{N} W_{i}(x) f_{h 1}\left(x_{\bar{V} i}\right) \\
& \cdot\left[V_{\alpha 1}^{(1)}\left(x_{\bar{V} i}\right) \alpha+V_{\alpha 2}^{(1)}\left(x_{\bar{V} i}\right) \alpha_{\mathrm{squ}}+\alpha^{\mathrm{T}} V_{\alpha 3}\left(x_{\bar{V} i}\right) \alpha\right. \\
& \left.+\alpha_{\mathrm{squ}}^{\mathrm{T}} V_{\alpha 4}\left(x_{\bar{V} i}\right) \alpha+\alpha_{\mathrm{squ}}^{\mathrm{T}} V_{\alpha 5}\left(x_{\bar{V} i}\right) \alpha_{\mathrm{squ}}\right] \\
= & h_{\alpha 1,1}^{(1)}(\bar{V}) \alpha+h_{\alpha 2,1}^{(1)}(\bar{V}) \alpha_{\mathrm{squ}}+\alpha^{T} h_{\alpha 3,1}(\bar{V}) \alpha \\
& +\alpha_{\mathrm{squ}}^{\mathrm{T}} h_{\alpha 4,1}(\bar{V}) \alpha+\alpha_{\mathrm{squ}}^{\mathrm{T}} h_{\alpha 5}(\bar{V}) \alpha,
\end{aligned}
$$

where

$$
\begin{aligned}
h_{\alpha 1,1}^{(1)}(\bar{V})= & r_{0} W^{T}(\bar{V}) F_{h 1} A_{c b}\left[F_{V 2} A_{c b} F_{V 3}\right. \\
& \left.+F_{V 4} A_{c b}\left(F_{h 1} A_{c b} F_{V 3}+F_{h 2}\right)\right] A_{c b} F_{\gamma 1}, \\
h_{\alpha 2,1}^{(1)}(\bar{V})= & r_{0} W^{\mathrm{T}}(\bar{V}) F_{h 1} A_{c b}\left(F_{V 2}+F_{V 4} A_{c b} F_{h 1}\right) A_{c b} F_{V 1}, \\
h_{\alpha 3,1}(\bar{V})= & \frac{1}{2}\left(\bar{V}-\bar{V}_{0}\right) r_{0} \\
& \cdot \sum_{j=0}^{N}\left[\sum_{i=0}^{N} W_{i}(x) W_{j}\left(x_{\bar{V} i}\right) f_{h 1}\left(x_{\bar{V} i}\right) f_{V 6}\left(x_{\bar{V} j}\right)\right] \\
& \cdot\left[\gamma_{\alpha 1}^{(0)}\left(x_{\bar{V} j}\right)\right]^{\mathrm{T}} \gamma_{\alpha 1}^{(0)}\left(x_{\bar{V} j}\right), \\
h_{\alpha 4,1}(\bar{V})= & \frac{1}{2}\left(\bar{V}-\bar{V}_{0}\right) \\
& \cdot \sum_{j=0}^{N}\left[\sum_{i=0}^{N} W_{i}(x) W_{j}\left(x_{\bar{V} i}\right) f_{h 1}\left(x_{\bar{V} i}\right) f_{V 5}\left(x_{\bar{V} j}\right)\right] \\
& \cdot I^{\mathrm{T}}(j) h_{\alpha 1}^{(0)}\left(x_{\bar{V} j}\right), \\
h_{\alpha 5}(\bar{V})= & \frac{1}{2}\left(\bar{V}-\bar{V}_{0}\right) \\
& \cdot \sum_{j=0}^{N}\left[\sum_{i=0}^{N} W_{i}(x) W_{j}\left(x_{\bar{V} i}\right) f_{h 1}\left(x_{\bar{V} i}\right) f_{V 5}\left(x_{\bar{V} j}\right)\right] \\
& \cdot I^{\mathrm{T}}(j) h_{\alpha 2}^{(0)}\left(x_{\bar{V} j}\right) .
\end{aligned}
$$

Similar to (91), the second term of (90) can be rewritten as

$$
\begin{aligned}
\Delta h_{2}^{(1)}(\bar{V})= & h_{\alpha 1,2}^{(1)}(\bar{V}) \alpha+h_{\alpha 2,2}^{(1)}(\bar{V}) \alpha_{\text {squ }}+\alpha^{\mathrm{T}} h_{\alpha 3,2}(\bar{V}) \alpha \\
& +\alpha_{\text {squ }}^{\mathrm{T}} h_{\alpha 4,2}(\bar{V}) \alpha,
\end{aligned}
$$

where

$$
\begin{aligned}
h_{\alpha 1,2}^{(1)}(\bar{V})= & r_{0} W^{\mathrm{T}}(\bar{V}) F_{h 2} A_{c b}\left(F_{\gamma 2} A_{c b} F_{V 3}+F_{\gamma 3}\right) A_{c b} F_{\gamma 1}, \\
h_{\alpha 2,2}^{(1)}(\bar{V})= & r_{0} W^{\mathrm{T}}(\bar{V}) F_{h 2} A_{c b} F_{\gamma 2} A_{c b} F_{V 1}, \\
h_{\alpha 3,2}(\bar{V})= & \frac{1}{2}\left(\bar{V}-\bar{V}_{0}\right) r_{0} \\
& \cdot \sum_{j=0}^{N}\left[\sum_{i=0}^{N} W_{i}(x) W_{j}\left(x_{\bar{V} i}\right) f_{h 2}\left(x_{\bar{V} i}\right) f_{\gamma 5}\left(x_{\bar{V} j}\right)\right] \\
& \cdot\left[\gamma_{\alpha 1}^{(0)}\left(x_{\bar{V} j}\right)\right]^{\mathrm{T}} \gamma_{\alpha 1}^{(0)}\left(x_{\bar{V} j}\right)+\frac{1}{2}\left(\bar{V}-\bar{V}_{0}\right) \\
& \cdot \sum_{j=0}^{N}\left[\sum_{i=0}^{N} W_{i}(x) W_{j}\left(x_{\bar{V} i}\right) f_{h 2}\left(x_{\bar{V} i}\right) f_{\gamma 4}\left(x_{\bar{V} j}\right)\right] \\
& \cdot\left[h_{\alpha 1}^{(0)}\left(x_{\bar{V} j}\right)\right]^{\mathrm{T}} I(j), \\
h_{\alpha 4,2}(\bar{V})= & \frac{1}{2}\left(\bar{V}^{2}-\bar{V}_{0}\right) \\
& \cdot \sum_{j=0}^{N}\left[\sum_{i=0}^{N} W_{i}(x) W_{j}\left(x_{\bar{V} i}\right) f_{h 2}\left(x_{\bar{V} i}\right) f_{\gamma 4}\left(x_{\bar{V} j}\right)\right] \\
& \cdot\left[h_{\alpha 2}^{(0)}\left(x_{\bar{V} j}\right)\right]^{\mathrm{T}} I(j) .
\end{aligned}
$$

Substituting (69) and (72) into the third term of (90), it can be expressed as

$$
\begin{aligned}
\Delta h_{3}^{(1)}(\bar{V})= & \frac{1}{2}\left(\bar{V}-\bar{V}_{0}\right) \frac{r_{0}}{V_{e}} \sum_{i=0}^{N} W_{i}(x) f_{h 3}\left(x_{\bar{V} i}\right) \\
& \cdot\left(V_{\alpha 1}^{(0)}\left(x_{\bar{V} i}\right) \alpha+V_{\alpha 2}^{(0)}\left(x_{\bar{V} i}\right) \alpha_{\mathrm{squ}}\right)\left(\gamma_{\alpha 1}^{(0)}\left(x_{\bar{V} i}\right) \alpha\right) \\
& +\frac{1}{2}\left(\bar{V}-\bar{V}_{0}\right) r_{0} \sum_{i=0}^{N} W_{i}(x) f_{h 4}\left(x_{\bar{V} i}\right)\left(\gamma_{\alpha 1}^{(0)}\left(x_{\bar{V} i}\right) \alpha\right)^{2} \\
= & \alpha^{\mathrm{T}} h_{\alpha 3,3}(\bar{V}) \alpha+\alpha_{\mathrm{squ}}^{\mathrm{T}} h_{\alpha 4,3}(\bar{V}) \alpha,
\end{aligned}
$$

where

$$
\begin{aligned}
h_{\alpha 3,3}(\bar{V})= & \frac{1}{2}\left(\bar{V}-\bar{V}_{0}\right) \frac{r_{0}}{V_{e}} \sum_{i=0}^{N} W_{i}(x) f_{h 3}\left(x_{\bar{V} i}\right) \\
& \cdot\left[V_{\alpha 1}^{(0)}\left(x_{\bar{V} i}\right)\right]^{\mathrm{T}} \gamma_{\alpha 1}^{(0)}\left(x_{\bar{V} i}\right)+\frac{1}{2}\left(\bar{V}-\bar{V}_{0}\right) r_{0} \\
& \cdot \sum_{i=0}^{N} W_{i}(x) f_{h 4}\left(x_{\bar{V} i}\right)\left[\gamma_{\alpha 1}^{(0)}\left(x_{\bar{V} i}\right)\right]^{\mathrm{T}} \gamma_{\alpha 1}^{(0)}\left(x_{\bar{V} i}\right),
\end{aligned}
$$

$$
\begin{aligned}
h_{\alpha 4,3}(\bar{V})= & \frac{1}{2}\left(\bar{V}-\bar{V}_{0}\right) \frac{r_{0}}{V_{e}} \sum_{i=0}^{N} W_{i}(x) f_{h 3}\left(x_{\bar{V} i}\right) \\
& \cdot\left[V_{\alpha 2}^{(0)}\left(x_{\bar{V} i}\right)\right]^{\mathrm{T}} \gamma_{\alpha 1}^{(0)}\left(x_{\bar{V} i}\right) .
\end{aligned}
$$


Therefore, combining (91), (93), and (95), the analytical solution to $\Delta h^{(1)}$ is expressed as

$$
\begin{aligned}
\Delta h^{(1)}(\bar{V})= & h_{\alpha 1}^{(1)}(\bar{V}) \alpha+h_{\alpha 2}^{(1)}(\bar{V}) \alpha_{\text {squ }}+\alpha^{\mathrm{T}} h_{\alpha 3}(\bar{V}) \alpha \\
& +\alpha_{\text {squ }}^{\mathrm{T}} h_{\alpha 4}(\bar{V}) \alpha+\alpha_{\text {squ }}^{\mathrm{T}} h_{\alpha 5}(\bar{V}) \alpha
\end{aligned}
$$

where

$$
\begin{aligned}
& h_{\alpha 1}^{(1)}(\bar{V})=h_{\alpha 1,1}^{(1)}(\bar{V})+h_{\alpha 1,2}^{(1)}(\bar{V}), \\
& h_{\alpha 2}^{(1)}(\bar{V})=h_{\alpha 2,1}^{(1)}(\bar{V})+h_{\alpha 2,2}^{(1)}(\bar{V}), \\
& h_{\alpha 3}(\bar{V})=h_{\alpha 3,1}(\bar{V})+h_{\alpha 3,2}(\bar{V})+h_{\alpha 3,3}(\bar{V}), \\
& h_{\alpha 4}(\bar{V})=h_{\alpha 4,1}(\bar{V})+h_{\alpha 4,2}(\bar{V})+h_{\alpha 4,3}(\bar{V}) .
\end{aligned}
$$

3.3.4. Full Analytical Solution to Trajectory with Arbitrary $A O A$. When the zero-order and first-order solutions are derived, the full analytical solutions to trajectory increment can be formulated as

$$
\begin{aligned}
\Delta x(\bar{V})= & X_{\alpha 1}(\bar{V}) \alpha+X_{\alpha 2}(\bar{V}) \alpha_{\text {squ }}+A^{\mathrm{T}} X_{\alpha 3}(\bar{V}) \alpha \\
& +A_{\text {squ }}^{\mathrm{T}} X_{\alpha 4}(\bar{V}) \alpha+A_{\text {squ }}^{\mathrm{T}} X_{\alpha 5}(\bar{V}) \alpha_{\text {squ }}
\end{aligned}
$$

where $A$ and $A_{\text {squ }}$ are the partitioned diagonal matrices expressed as (100), respectively.

$$
A=\operatorname{diag}\{\alpha, \alpha, \alpha\}, A_{\text {squ }}=\operatorname{diag}\left\{\alpha_{\text {squ }}, \alpha_{\text {squ }}, \alpha_{\text {squ }}\right\} .
$$

The coefficients are

$$
X_{\alpha i}(\bar{V})=\left[V_{\alpha i}^{\mathrm{T}}(\bar{V}), \gamma_{\alpha i}^{\mathrm{T}}(\bar{V}), h_{\alpha i}^{\mathrm{T}}(\bar{V})\right]^{\mathrm{T}}, \quad i=1,2,3,4,5,
$$

and each coefficient is determined by the sum of the zeroth-order and first-order terms.

Then, the analytical solution to ascent trajectory can be expressed as

$$
\begin{aligned}
x= & x_{b}(\bar{V})+X_{\alpha 1}(\bar{V}) \alpha+X_{\alpha 2}(\bar{V}) \alpha_{\text {squ }}+A^{\mathrm{T}} X_{\alpha 3}(\bar{V}) \alpha \\
& +A_{\text {squ }}^{\mathrm{T}} X_{\alpha 4}(\bar{V}) \alpha+A_{\text {squ }}^{\mathrm{T}} X_{\alpha 5}(\bar{V}) \alpha_{\text {squ }},
\end{aligned}
$$

where $x_{b}(\bar{V})$ is the analytical solution to the trajectory with zero AOA, which is expressed as

$$
x_{b}(\bar{V})=\left[\begin{array}{lll}
V_{b}^{(0)}+V_{b}^{(1)}, & \gamma_{b}^{(0)}+\gamma_{b}^{(1)}, \quad h_{b}^{(0)}+h_{b}^{(1)}
\end{array}\right]^{\mathrm{T}} .
$$

It should be noted that the analytical solution includes the high order of AOA which leads to higher accuracy for states in all region than that only first order of AOA is considered.

\section{Optimal AOA Correction with Terminal Constraint}

In the last section, the analytical solutions to ascent trajectory with arbitrary AOA profile are derived. The optimal AOA correction can be developed to remove terminal errors. Now, let us consider the nonlinear optimal control problem with the performance index expressed as (4) which subjects to dynamical constraints presented in (1) and the hard terminal constraints (3).

The dynamic constraints presented in (1) can be transformed into the following algebraic constraints using the analytic solutions previously derived:

$$
\begin{aligned}
x\left(t_{f}\right)= & x_{b}\left(\bar{V}_{f}\right)+X_{\alpha 1}\left(\bar{V}_{f}\right) \alpha+X_{\alpha 2}\left(\bar{V}_{f}\right) \alpha_{\text {squ }}+A^{\mathrm{T}} X_{\alpha 3}\left(\bar{V}_{f}\right) \alpha \\
& +A_{\text {squ }}^{\mathrm{T}} X_{\alpha 4}\left(\bar{V}_{f}\right) \alpha+A_{\text {squ }}^{\mathrm{T}} X_{\alpha 5}\left(\bar{V}_{f}\right) \alpha_{\text {squ }},
\end{aligned}
$$

where $\bar{V}_{f}$ is the value of $\bar{V}$ at the terminal time. Then, substituting (104) into (3), the terminal constraint functions can be converted to the functions about the AOA vector, $\alpha$ :

$$
\Phi(\alpha)=\psi\left(x\left(t_{f}\right)\right)=0
$$

Furthermore, using Chebyshev polynomial approximation, the performance index expressed in (4) can also be converted into

$$
\begin{aligned}
J= & \frac{1}{2}\left[T \exp \left(\bar{V}_{0}\right)\right]^{-n+1} \int_{\bar{V}_{0}}^{\bar{V}_{f}} \frac{\alpha^{2}\left(x_{\bar{V}}\right) \exp \left(-x_{\bar{V}}\right)}{\left[\exp \left(-x_{\bar{V}}\right)-\exp \left(-\bar{V}_{f}\right)\right]^{n}} d x_{\bar{V}} \\
= & \frac{1}{4}\left(\bar{V}_{f}-\bar{V}_{0}\right)\left[T \exp \left(\bar{V}_{0}\right)\right]^{-n+1} \\
& \cdot \sum_{i=0}^{N} \frac{W_{i} \exp \left(-x_{\bar{V} i}\right) \alpha^{2}\left(x_{\bar{V} i}\right)}{\left.\exp \left(-x_{\bar{V} i}\right)-\exp \left(-\bar{V}_{f}\right)\right]^{n}} \\
= & \frac{1}{2} \alpha^{\mathrm{T}} Q \alpha,
\end{aligned}
$$

where $Q \in R^{N \times N}$ is a positive definite diagonal matrix whose elements along the diagonal are

$$
\begin{aligned}
Q_{i}= & \frac{1}{2}\left(\bar{V}_{f}-\bar{V}_{0}\right)\left[T \exp \left(\bar{V}_{0}\right)\right]^{-n+1} \\
& \cdot \frac{W_{i} \exp \left(-x_{\bar{V} i}\right)}{\left[\exp \left(-x_{\bar{V} i}\right)-\exp \left(-\bar{V}_{f}\right)\right]^{n}}, \quad i=0,1, \cdots, N .
\end{aligned}
$$

Finally, the nonlinear optimal control problem is transformed into a nonlinear constrained optimization problem, of which the performance index and constraints are (106) and (104), respectively. And the optimal variables are the $\mathrm{AOA}$ at the interpolation points, $\alpha\left(x_{\bar{V}_{i}}\right)(i=0,1, \cdots, N)$. The nonlinear constrained optimization problem can further be transformed into a series of quadratic programming problems if linearization is applied for the terminal constraints. The optimal variables are obtained by iteratively 


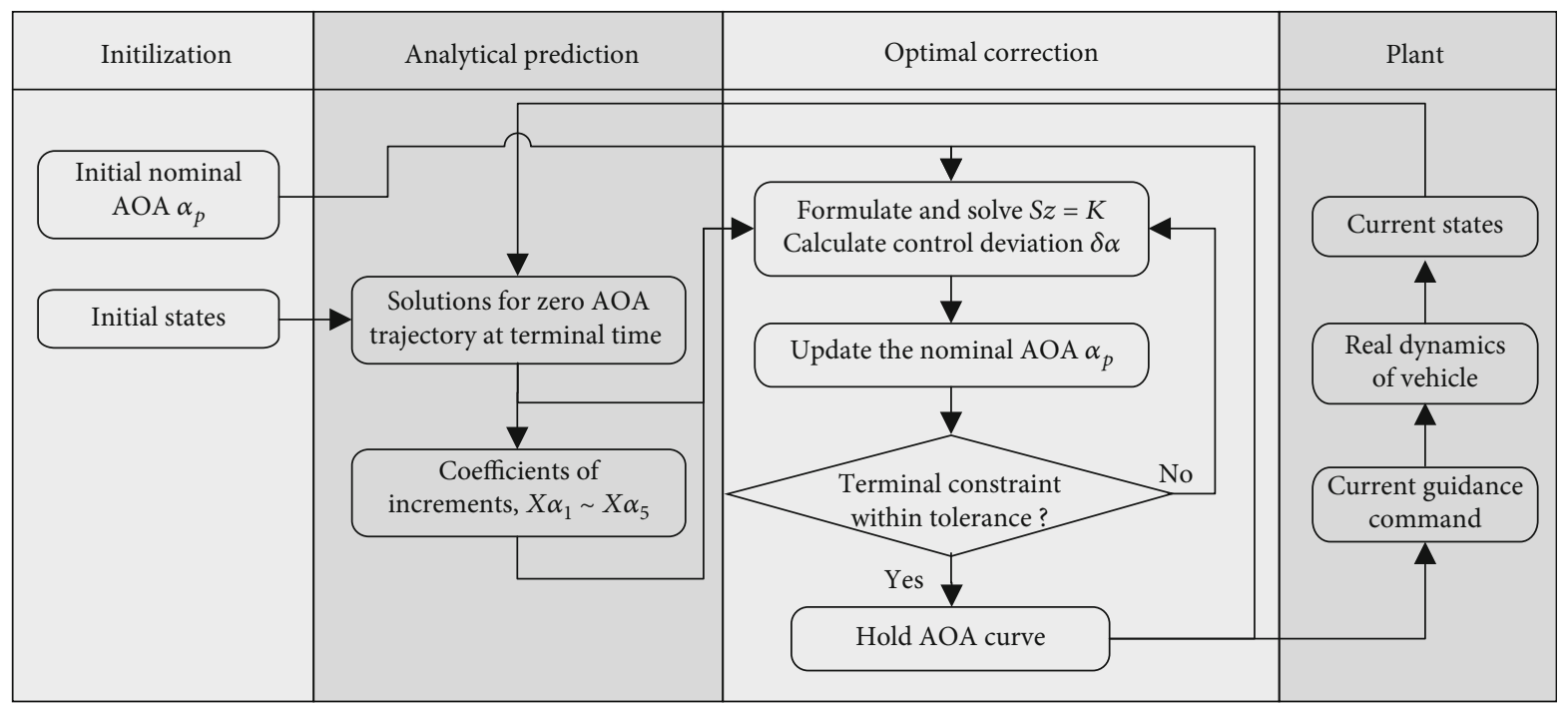

Figure 3: Flow chart for implementing the proposed guidance law.

solving this series of quadratic programming problems. The derivation process is presented as follows.

Expanding (105) in Taylor series around the trajectory with the nominal AOA curve, $\alpha_{p}$. Then, a set of linear terminal constraints (108) using the deviations from the nominal $\mathrm{AOA}$ as the independent variables are formulated by neglecting the higher order terms:

$$
\psi\left(x_{p f}\right)+\frac{\partial \psi}{\partial x_{f}}\left(x_{p f}\right) \frac{\partial \delta x_{f}}{\partial \alpha}\left(\alpha_{p}\right) \delta \alpha=0
$$

where $\alpha_{p}$ is normal AOA vector whose elements are the value of nominal AOA profile at interpolating points, i.e.,

$$
\alpha_{p}=\left[\alpha_{p}\left(x_{\bar{V} 0}\right), \alpha_{p}\left(x_{\bar{V} 1}\right), \cdots, \alpha_{p}\left(x_{\bar{V} N}\right)\right]^{\mathrm{T}} \text {. }
$$

$\delta \alpha=\alpha-\alpha_{p}$ is the deviation of the real AOA from the nominal AOA; $\partial \psi / \partial x_{f}(\cdot)$ is the partial derivative of the terminal constraint function $\psi(\cdot)$ with respect to the terminal state $x_{f}$; and $\partial \delta x_{f} / \partial \alpha(\cdot)$ is the partial derivative of the terminal state with respect to the vector of AOA at the interpolation points, $\alpha$, which can be expressed as

$$
\begin{aligned}
\frac{\partial \delta x_{f}}{\partial \alpha}(\alpha)= & X_{\alpha 1}+2 X_{\alpha 2} \tilde{\alpha}+A^{\mathrm{T}}\left(X_{\alpha 3}+\tilde{X}_{\alpha 3}\right)+A_{\mathrm{squ}}^{\mathrm{T}} X_{\alpha 4} \\
& +2 A^{\mathrm{T}} \tilde{X}_{\alpha 4} \tilde{\alpha}+2 A_{\mathrm{squ}}^{\mathrm{T}}\left(X_{\alpha 5}+\tilde{X}_{\alpha 5}\right) \tilde{\alpha}
\end{aligned}
$$

where $\tilde{\alpha}=\operatorname{diag}\{\alpha\}$ and $\tilde{X}_{\alpha i}=\left[V_{\alpha i}, \gamma_{\alpha i}, h_{\alpha i}\right]^{\mathrm{T}}, i=3,4,5 . x_{p f}$ is the terminal value of trajectory with the nominal AOA profile:

$$
\begin{aligned}
x_{p f}= & x_{b}\left(\bar{V}_{f}\right)+X_{\alpha 1}\left(\bar{V}_{f}\right) \alpha_{p}+X_{\alpha 2}\left(\bar{V}_{f}\right) \alpha_{p, \mathrm{squ}}+A_{p}^{\mathrm{T}} X_{\alpha 3}\left(\bar{V}_{f}\right) \alpha_{p} \\
& +A_{p, \mathrm{squ}}^{\mathrm{T}} X_{\alpha 4}\left(\bar{V}_{f}\right) \alpha_{p}+A_{p, \mathrm{squ}}^{\mathrm{T}} X_{\alpha 5}\left(\bar{V}_{f}\right) \alpha_{p, \mathrm{squ}} .
\end{aligned}
$$

Table 2: Angle of Attack curves.

\begin{tabular}{lc}
\hline Case & AOA curve \\
\hline 1 & $\alpha(t)=\alpha_{0}\left[6.25\left(t / t_{f}-0.6\right)^{2}-1\right]$ \\
2 & $\alpha(t)=\alpha_{0}\left(t / t_{f}-1\right)^{2}$ \\
3 & $\alpha(t)=\alpha_{0}\left(1-2 t / t_{f}\right)$ \\
\hline
\end{tabular}

As for the performance index (106), using the $\delta \alpha$ as the optimal variables, it can also be rewritten as

$$
J=\frac{1}{2} \alpha_{p}^{\mathrm{T}} Q \alpha_{p}+\alpha_{p}^{\mathrm{T}} Q \delta \alpha+\frac{1}{2} \delta \alpha^{\mathrm{T}} Q \delta \alpha
$$

Considering the terminal constraints, the augmented performance index is

$$
\bar{J}=J+v^{\mathrm{T}}\left[\psi\left(x_{p f}\right)+\frac{\partial \psi}{\partial x_{f}}\left(x_{p f}\right) \frac{\partial \delta x_{f}}{\partial \alpha}\left(\alpha_{p}\right) \delta \alpha\right],
$$

where $v \in R^{S}$ is the Lagrange multiplier vector. According to numerical optimization theory, the corresponding KKT conditions are

$$
\left\{\begin{array}{l}
\frac{\partial \bar{J}}{\partial \delta \alpha}=Q\left(\alpha_{p}+\delta \alpha\right)+\left[\frac{\partial \psi}{\partial x_{f}}\left(x_{p f}\right) \frac{\partial \delta x_{f}}{\partial \alpha}\left(\alpha_{p}\right)\right]^{\mathrm{T}} v=0, \\
\frac{\partial \bar{J}}{\partial v}=\psi\left(x_{p f}\right)+\dot{\psi}\left(x_{p f}\right) \delta \dot{x}_{f}\left(\alpha_{p}\right) \delta \alpha=0 .
\end{array}\right.
$$

It is easy to find that the KKT conditions are a set of linear algebraic equations which can be expressed as

$$
S z=K,
$$




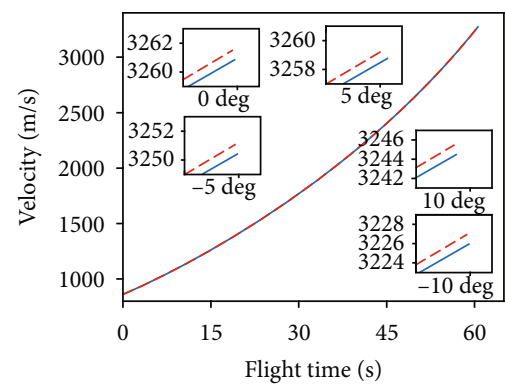

- Numerical solution

--- Analytical solution

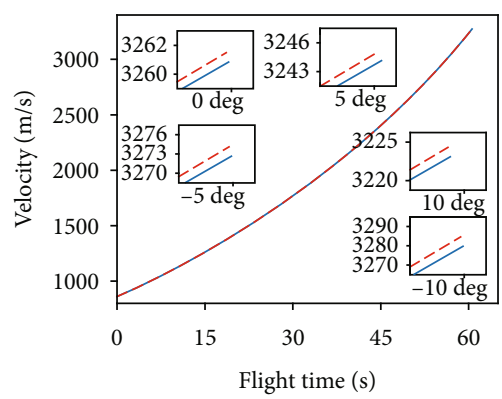

- Numerical solution

--- Analytical solution

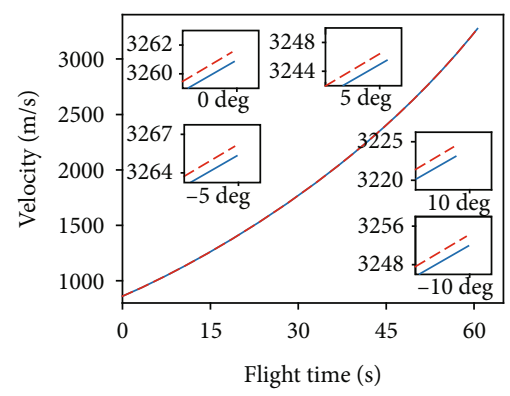

- Numerical solution

- - - Analytical solution

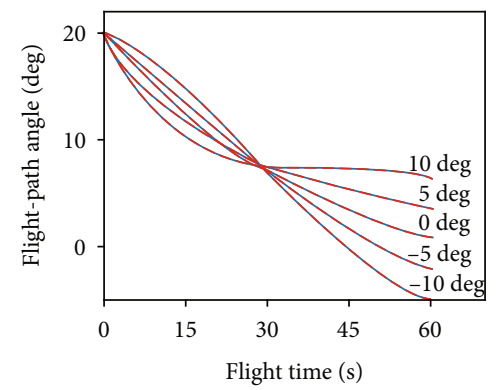

AOA curve 1

(a) AOA curve 1
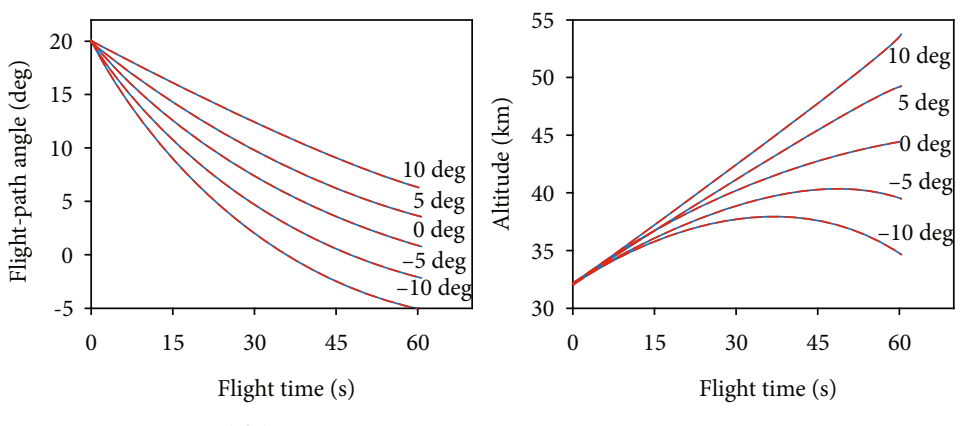

(b) AOA curve 2
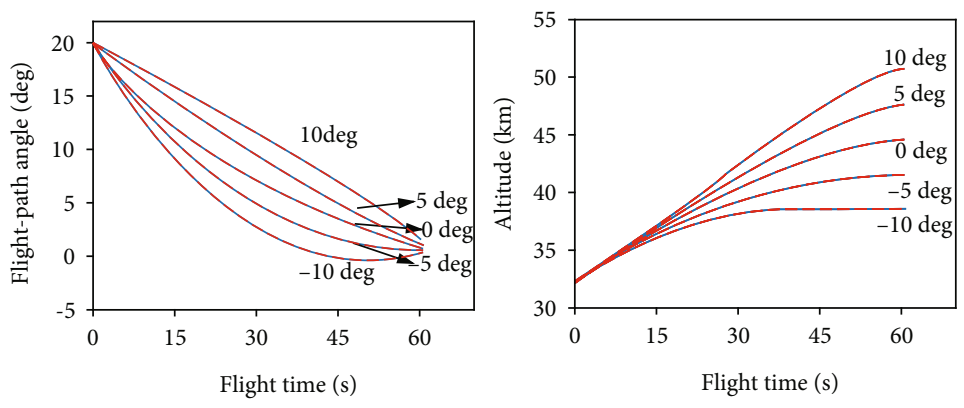

AOA curve 3

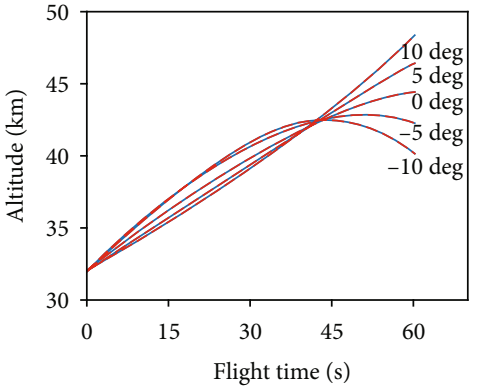


TABLE 3: Comparison of the analytical solutions and numerical solutions.

\begin{tabular}{|c|c|c|c|c|c|}
\hline Case & Methods & $V_{f}(\mathrm{~m} / \mathrm{s})$ & $\gamma_{f}(\operatorname{deg})$ & $h_{f}(\mathrm{~m})$ & Computing time (s) \\
\hline \multirow{3}{*}{1} & Numerical solutions & 3225.57 & 6.4383 & 48217.10 & 0.0230 \\
\hline & Analytical solutions & 3227.14 & 6.4492 & 48279.28 & $8.03 E-04$ \\
\hline & Relative errors & $0.067 \%$ & $0.079 \%$ & $0.38 \%$ & - \\
\hline \multirow{3}{*}{2} & Numerical solutions & 3272.49 & -5.0089 & 34662.20 & 0.0267 \\
\hline & Analytical solutions & 3288.19 & -4.9948 & 34929.09 & $7.16 E-04$ \\
\hline & Relative errors & $0.48 \%$ & $-0.28 \%$ & $0.77 \%$ & - \\
\hline \multirow{3}{*}{3} & Numerical solutions & 3250.87 & 0.5822 & 38319.76 & 0.0234 \\
\hline & Analytical solutions & 3259.15 & 0.4528 & 38297.28 & $7.86 E-04$ \\
\hline & Relative errors & $0.35 \%$ & $0.67 \%$ & $-0.36 \%$ & - \\
\hline
\end{tabular}

is needed to calculate those coefficients at each guidance period. Additionally, the value of AOA at the arbitrary time $t$ can be approximated by Chebyshev interpolating polynomial:

$$
\alpha(t)=\sum_{i=0}^{N} \alpha\left(x_{\bar{V} i}\right) L_{i}\left(x_{t}\right)
$$

where $x_{t}$ is the value of $x$ at the time $t$, which can be obtained by

$$
x_{t}=\frac{\bar{V}-\bar{V}_{2}}{\bar{V}_{1}}=-\frac{2}{\bar{V}_{f}-\bar{V}_{0}} \ln \left[1-\frac{t-t_{0}}{T}\right]-1 \text {. }
$$

\section{Implementation of Ascent Guidance Law}

In this section, an ascent guidance law with terminal constraints is proposed, in which the current AOA command is generated by iteratively solving the nonlinear optimal control problem using the analytical trajectory prediction. The procedure for implementing the proposed guidance law is included in the flow chart in Figure 3 and summarized in detail as follows.

(1) In the initialization, set initial parameters and select zero AOA as the initial nominal AOA $\alpha_{p}$

(2) Calculate the analytical solutions to $x_{b f}$ and the coefficients $X_{\alpha 1} \sim X_{\alpha 5}$ using the current states

(3) Apply the method of linearization around the nominal AOA $\alpha_{p}$ to formulate the matrices $S$ and $K$, and calculate control deviation $\delta \alpha$ by solving linear algebraic equations

(4) Generate the new nominal AOA, and repeat step 3 until $\left\|\psi\left(x\left(t_{f}\right)\right)\right\|$ is within the prescribed tolerance, then go to Step 5

(5) Update the current guidance command $\alpha\left(t_{0}\right)$ by (118). Then, go to next step
TABLE 4: Terminal constraints for different cases.

\begin{tabular}{lcccc}
\hline Constraints & Case 1 & Case 2 & Case 3 & Case 4 \\
\hline$\gamma_{f c}(\mathrm{deg})$ & 0 & 0 & 5 & 5 \\
$h_{f c}(\mathrm{~km})$ & 40 & 45 & 45 & 40 \\
\hline
\end{tabular}

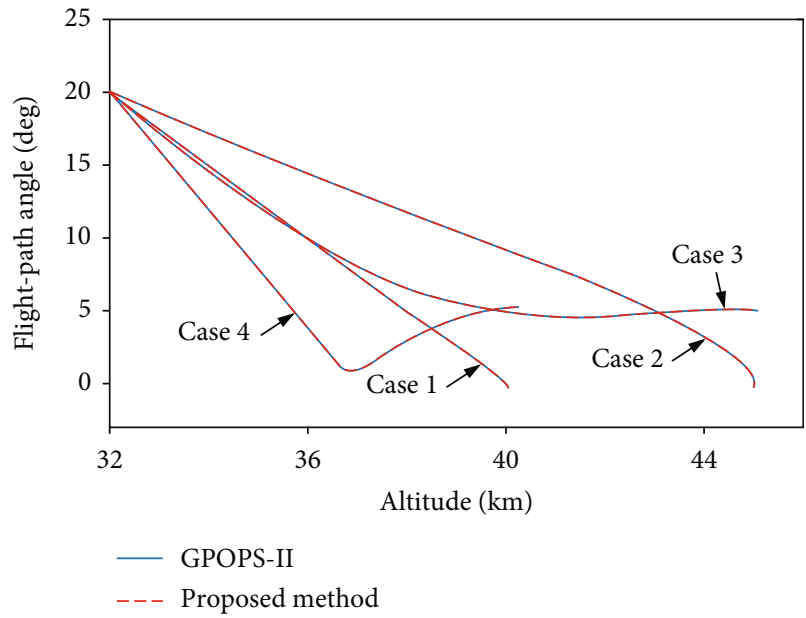

Figure 5: Altitude vs. flight-path angle histories.

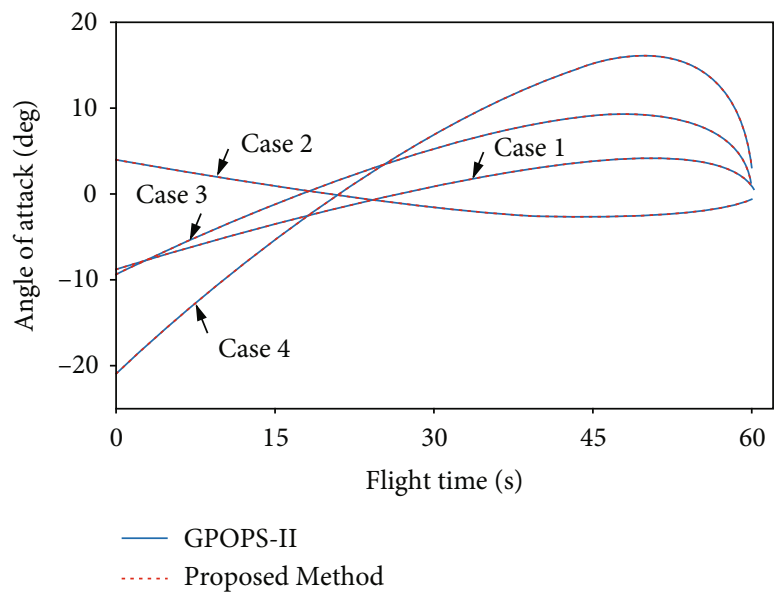

Figure 6: AOA profiles. 
TABLE 5: Terminal relative errors and computation times for different cases.

\begin{tabular}{lcccc}
\hline Case & Method & Flight-path angle error & Altitude error & Computing time (s) \\
\hline \multirow{2}{*}{1} & GPOPS-II & $0.0025 \%$ & $-0.0053 \%$ & 0.171 \\
& Proposed method & $0.063 \%$ & $-0.042 \%$ & $2.2 \mathrm{E}-03$ \\
\hline \multirow{2}{*}{3} & GPOPS-II & $-0.0036 \%$ & $0.0042 \%$ & 0.119 \\
& Proposed method & $-0.069 \%$ & $-0.0032 \%$ & $2.5 \mathrm{E}-03$ \\
\hline \multirow{2}{*}{4} & GPOPS-II & $0.0044 \%$ & $0.15 \%$ & 2.280 \\
& Proposed method & $-0.078 \%$ & $-0.0038 \%$ & 0.6772 \\
& GPOPS-II & $0.0034 \%$ & $-2.97 \%$ & $3.0 \mathrm{E}-03$ \\
\hline
\end{tabular}

(6) Apply $\alpha\left(t_{0}\right)$ to the real vehicle and then return Step 2. Also, the AOA obtained at Step 4 is used as the initial nominal AOA for the next guidance step

Different from the traditional guidance laws, the proposed guidance does not need to conduct any offline planning for specific mission. Therefore, this guidance can handle various urgent tasks. Besides, it can significantly reduce the computational load compared to the method based on the numerical solutions because the latter needs to conduct thousands of numerical trajectory integrations for iterations in practice.

\section{Simulation and Analysis}

In this section, a comparison with the numerical integration is provided to verified the accuracy and efficiency of the analytical trajectory prediction. Then, several nominal cases and Monte Carlo simulations with dispersions and uncertainties are carried out to evaluate the performance and robustness of the proposed method. In which, a typical launch vehicle described in previous section is used. All programs are implemented on a personal computer with $2.6 \mathrm{GHz}$ processor and 2020a MATLAB environment.

6.1. Accuracy Verification for Analytical Solution with Arbitrary AOA. The accuracy of proposed guidance law is highly dependent with the analytical trajectory prediction, which provides the nominal trajectory for Taylor expansion. Therefore, an accuracy verification for analytical solution is provided in this subsection. For all simulations, the initial conditions are set to $V_{0}=875 \mathrm{~m} / \mathrm{s}, \gamma_{0}=20 \mathrm{deg}$, and $h_{0}=$ $32 \mathrm{~km}$. And three types of AOA curve listed in Table 2 are considered.

Firstly, the cases of three types of AOA curve with $\alpha_{0}=$ $10^{\circ}, 5^{\circ}, 0^{\circ},-5^{\circ},-10^{\circ}$ are simulated. Figure 4 shows the time histories of velocity, flight-path angle, and altitude for the different AOA curves. Obviously, the errors between the analytical solutions and numerical solutions are very small. In order to provide the quantitative description on the errors, the terminal states for analytical solutions and numerical solutions of $\alpha_{0}=-10^{\circ}$ are presented in Table 3 . Table 3 shows that the relative errors are all less than $1 \%$, which verifies the accuracy of analytical solutions. It is noted
TABLE 6: Initial conditions and terminal constraints for different cases.

\begin{tabular}{lcccc}
\hline Case & $\gamma_{0}(\mathrm{deg})$ & $h_{0}(\mathrm{~km})$ & $\gamma_{f c}(\mathrm{deg})$ & $h_{f c}(\mathrm{~km})$ \\
\hline 1 & 20 & 32 & 0 & 40 \\
2 & 20 & 32 & 0 & 45 \\
3 & 20 & 32 & 5 & 40 \\
4 & 20 & 32 & 5 & 45 \\
5 & 15 & 37 & 0 & 40 \\
6 & 25 & 37 & 0 & 40 \\
7 & 15 & 27 & 0 & 40 \\
8 & 25 & 27 & 0 & 40 \\
\hline
\end{tabular}

that the accuracy will be further improved as the total flight time decreases.

Besides, the computing time of the analytical and numerical solutions obtained by fourth-order Runge-Kutta integral with the step length of $0.1 \mathrm{~s}$ is presented in Table 3. The results indicate that the computing time of the analytical solutions is less than $1 \mathrm{~ms}$, which is about $3 \%$ of that of the numerical solutions. Obviously, the computational load of the trajectory planning method based on the numerical solutions is heavy because thousands of numerical trajectory integrations in practice are needed. However, if the analytical solutions are applied to trajectory planning, only one calculation is needed so that the consumed time will be greatly reduced. Conclusively, the analytical trajectory prediction has high accuracy and efficiency.

6.2. Nominal Case with Different Terminal Constraints. In this subsection, optimality and applicability of the proposed guidance law are evaluated by several different terminal constraints. It is noted that the initial conditions are the same as the last subsection and the coefficient $n$ is selected to be 0.5 .

6.2.1. Optimality Verification. In this subsection, the optimality of the proposed guidance law is verified by a comparison with the results generated by the world famous nonlinear optimal control solver software, GPOPS-II [41, 42]. There are four cases of terminal constraint which are listed in Table 4 are considered. It should be noted that all the results in this subsection are generated by open-loop optimal guidance. 

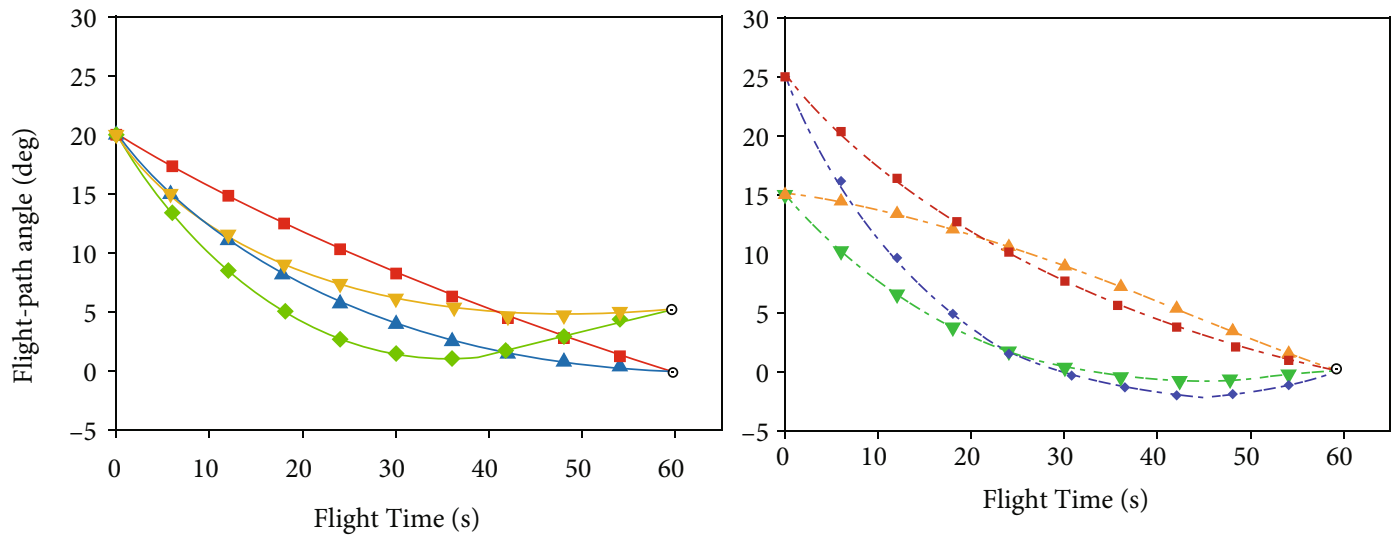

Flight-path angle histories

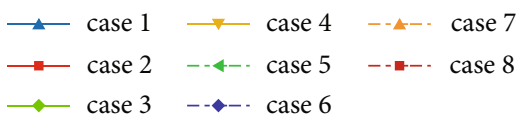

(a) Flight-path angle histories
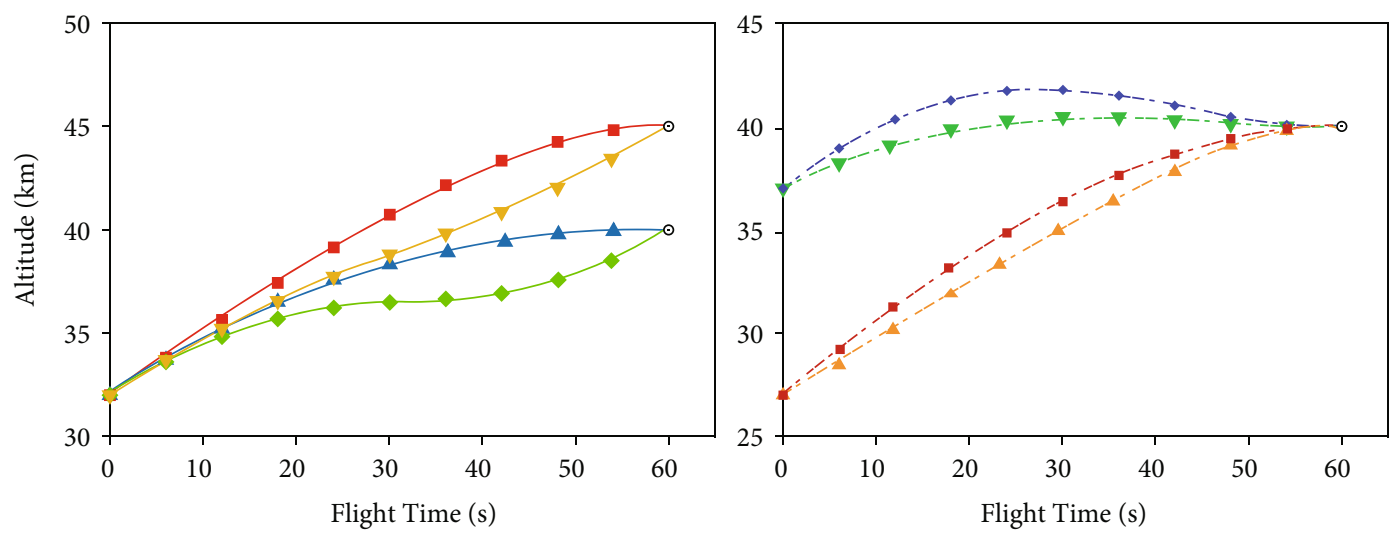

Altitude histories

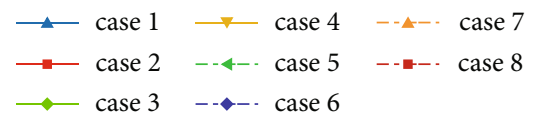

(b) Altitude histories

Figure 7: Flight-path angle and altitude histories for different cases.

TABle 7: Terminal errors with close-loop guidance.

\begin{tabular}{lccccc}
\hline Case & Flight-path angle error $(\mathrm{deg})$ & Altitude error $(\mathrm{m})$ & Case & Flight-path angle error $(\mathrm{deg})$ & Altitude error $(\mathrm{m})$ \\
\hline 1 & $-1.15 E-04$ & $1.71 E-03$ & 5 & $-1.7930 e-05$ & $1.9401 e-04$ \\
2 & $-2.04 E-04$ & $-4.94 E-04$ & 6 & $4.2814 e-05$ & $8.8906 e-04$ \\
3 & $-8.29 E-05$ & $-3.28 E-04$ & 7 & $1.0245 e-04$ & $1.5770 e-04$ \\
4 & $1.04 E-04$ & $6.61 E-05$ & 8 & $2.7622 e-05$ & $2.6255 e-05$ \\
\hline
\end{tabular}

Figures 5 and 6 depict the altitude vs. flight-path angle histories and AOA profiles obtained by the two methods, respectively. It is obvious that the all the curves are the same, which implies that the proposed method is capable of providing the optimal trajectory. The terminal errors of both methods for different cases are presented in Table 5. It can be seen from the table that although the terminal errors of the proposed method are slightly larger than that of GPOPS-II, it is still quite small, just less than $0.2 \%$ except case 4 . In case 4 , the terminal relative errors are a little large that about $3 \%$. This is because the AOA is larger than the other three cases, which results in the relatively larger errors 

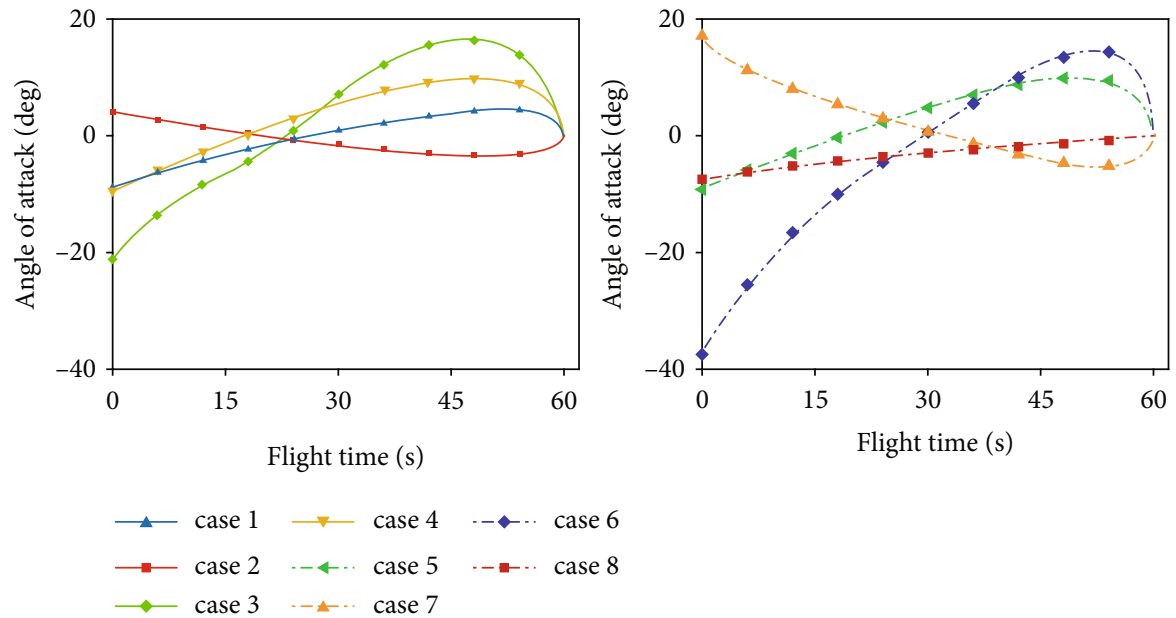

FIgURE 8: AOA profiles for different cases.

for the analytical integration. The errors absolutely met the requirement of the online guidance. Moreover, the accuracy will be improved as the time-to-go decreases.

Table 5 also lists the computing time of both methods. Though the accuracy of the proposed method is slightly lower than that of GPOPS-II, its computing time is less than $3 \mathrm{~ms}$, which is much less than that of GPOPS-II.

6.2.2. Applicability of Different Cases. In this subsection, the applicability of the proposed guidance law is tested by different initial conditions and terminal constraints, which are listed in Table 6. All the cases can be divided into two groups. The first one is cases 1-4, in which the initial flight-path angle and altitude are same and the terminal constraints are different. And the second one is cases $5-8$, in which the initial flight-path angle and altitude are different but the terminal constraints are same. Noted that the initial velocity is the same and set to $875 \mathrm{~m} / \mathrm{s}^{2}$ in all these cases. In these simulations, the real thrust of vehicle is regarded as the expression of (5), and the aerodynamic coefficients, $C_{l}^{\alpha}, C_{d 0}$, and $C_{d}^{\alpha}$, are approximated by the polynomials of Mach number as shown in (7). Additionally, International Standard Atmosphere (ISA) mode, in which the density and pressure are the piecewise functions of altitude, is adopted.

The flight-path angle and altitude histories are depicted in Figure 7. It is obvious that the proposed guidance is able to guide the vehicle to the desired terminal states with high accuracy. The terminal errors are presented in Table 7 . The maximum error for all cases is $2.04 E-04$ deg for fightpath angle and $8.8906 e-04 \mathrm{~m}$ for altitude. It is very small and meets the requirements on the subsequent successful handover. Note that the flight-path angle curves for cases 1 and 2 are monotonous, but the ones for cases 3 and 4 decrease at the beginning and then increase at the end. The reason is that the terminal altitude constrains for cases 3 and 4 are relatively low so that the flight-path angles need to decrease at the beginning so as to meet the altitude constraints. That is consistent with the requirement of the energy management. As for cases 5 and 6, the altitude increases at the beginning and then decreases at the end.

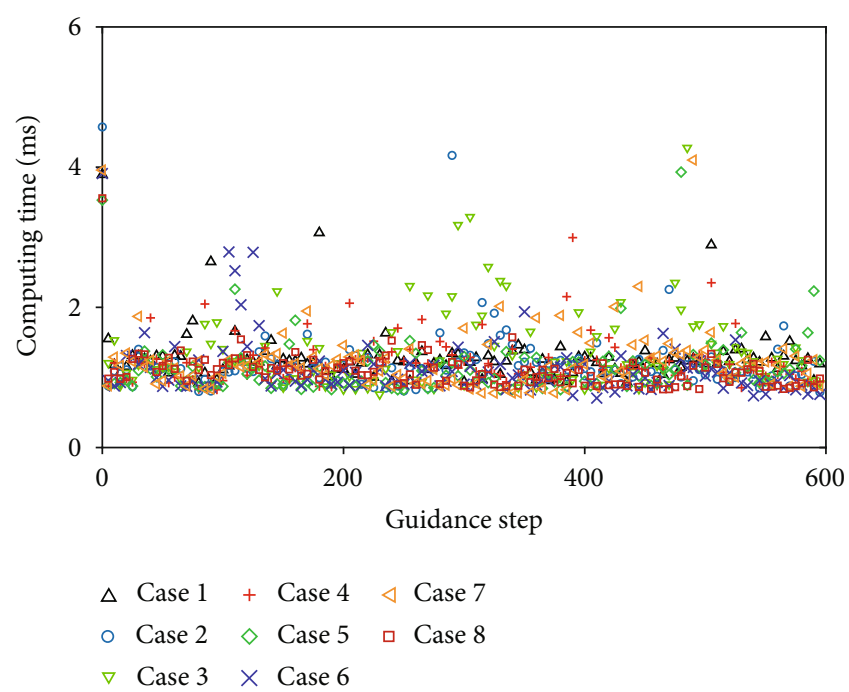

Figure 9: Consuming time at each guidance period.

TABle 8: The dispersion parameters of the Monte Carlo simulations.

\begin{tabular}{lccccc}
\hline Variables & $3 \sigma$ & Distribution & Variables & $3 \sigma$ & Distribution \\
\hline$\delta P_{0}$ & $15 \%$ & Gaussian & $k_{\delta P}^{h}$ & $15 \%$ & Gaussian \\
$\delta C_{l 0}$ & $15 \%$ & Gaussian & $\delta C_{d 0}$ & $15 \%$ & Gaussian \\
$k_{\delta C l}^{\mathrm{Ma}}$ & $30 \%$ & Gaussian & $k_{\delta C d}^{\mathrm{Ma}}$ & $30 \%$ & Gaussian \\
$k_{\delta C l}^{\alpha}$ & $45 \%$ & Gaussian & $k_{\delta C d}^{\alpha}$ & $45 \%$ & Gaussian \\
$V_{\text {wind }}$ & $300 \mathrm{~m} / \mathrm{s}$ & Gaussian & $\delta \rho$ & $30 \%$ & Gaussian \\
\hline
\end{tabular}

This is because the initial altitudes for these two case are large; the vehicle needs to climb and then descend to meet the terminal altitude constraints.

The AOA profiles obtained by proposed guidance are presented in Figure 8. Obviously, the terminal AOA for all cases converge to zero, which result from the weighted 


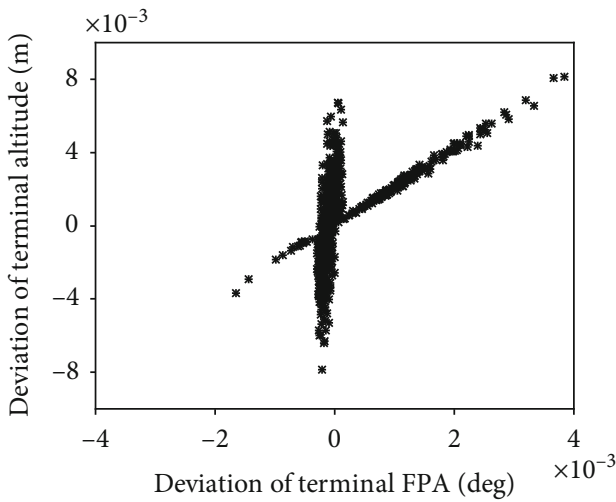

Case 1

(a) Case 1

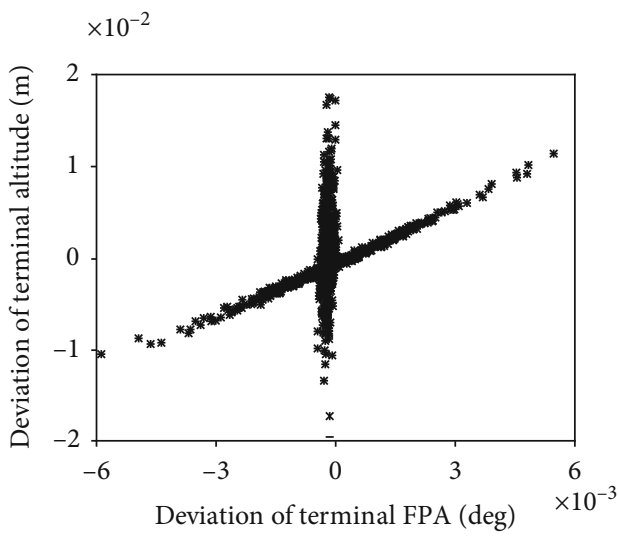

Case 3

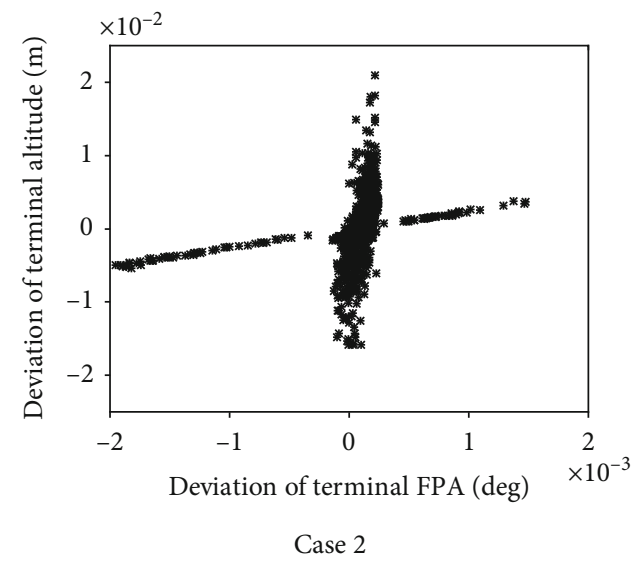

(b) Case 2

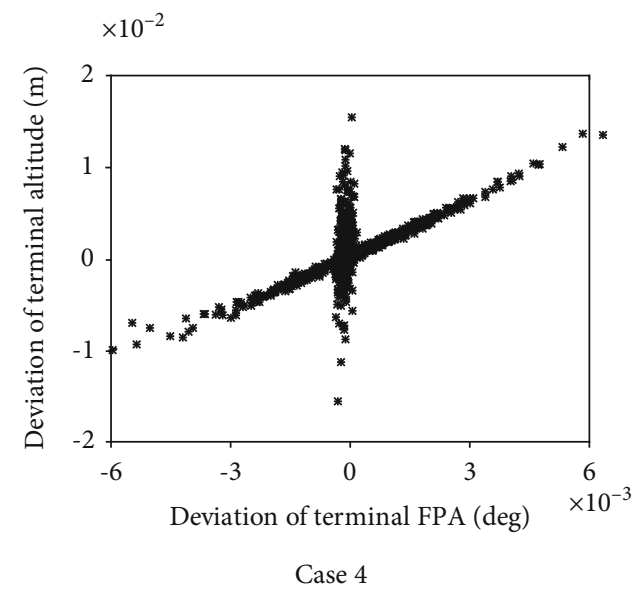

(d) Case 4

Figure 10: Terminal deviations of the Monte Carlo simulations.

squared sum performance index whose denominator is flight time to go. Additionally, all AOA profiles vary smoothly and its maximal value happens at the beginning. Moreover, as seen from Figure 9, let us focus on the consuming time for the proposed method at each guidance period. It is apparent that most of them distribute from $1 \mathrm{~ms}$ to $3 \mathrm{~ms}$, and its maximum is only $5 \mathrm{~ms}$. Therefore, the proposed guidance law has high computational efficiency and guidance accuracy.

6.3. Monte Carlo Simulations. In this subsection, 1000 times Monte Carlo simulations are performed to further verify the robustness of the guidance law. Dispersions and uncertainties including aerodynamics error, atmospheric uncertainties, thrust uncertainties, and wind interference are fully considered. In these cases, the percentage deviations of the lift and drag coefficients typically vary with Mach number and AOA. And the percentage deviations of the thrust typically vary with altitude. For simplicity, the linear dispersion model is used in the simulations.

$$
\delta P=\delta P_{0}+\frac{k_{\delta P}^{h}(91000-h)}{91000},
$$

TABle 9: The statistics on the terminal deviations of the Monte Carlo simulations.

\begin{tabular}{lcccc}
\hline Case & Mean $\delta \gamma_{f}(\mathrm{deg})$ & Mean $\delta h_{f}(\mathrm{~m})$ & Std $\delta \gamma_{f}(\mathrm{deg})$ & $\operatorname{Std} \delta h_{f}(\mathrm{~m})$ \\
\hline 1 & $4.90 E-04$ & $1.28 E-03$ & $9.36 E-05$ & $8.86 E-04$ \\
2 & $-7.68 E-04$ & $-2.47 E-04$ & $9.58 E-05$ & $3.86 E-03$ \\
3 & $-2.91 E-05$ & $1.02 E-03$ & $1.73 E-04$ & $4.62 E-03$ \\
4 & $2.61 E-05$ & $7.10 E-04$ & $1.62 E-04$ & $3.4 E-03$ \\
\hline
\end{tabular}

$$
\begin{aligned}
& \delta C_{l}=\delta C_{l 0}+k_{\delta C l}^{\mathrm{Ma}} \frac{M a-7}{8}+k_{\delta C l}^{\alpha} \frac{18}{\pi}\left(\alpha-\frac{\pi}{18}\right), \\
& \delta C_{d}=\delta C_{d 0}+k_{\delta C d}^{\mathrm{Ma}} \frac{M a-7}{8}+k_{\delta C d}^{\alpha} \frac{18}{\pi}\left(\alpha-\frac{\pi}{18}\right) .
\end{aligned}
$$

Dispersion parameters used in simulations are listed in Table $8 . V_{\text {Wind }}$ represents the wind speed, and $\delta \rho$ represents the percentage deviation of the atmospheric density. The first four cases listed in Table 6 are simulated in this subsection.

The dispersion of terminal states are presented in Figure 10. The terminal altitude errors are mostly near 
$0.01 \mathrm{~m}$, and the maximum is no more than $0.02 \mathrm{~m}$. The terminal flight-path angle errors are all within $6 \times 10^{-3} \mathrm{deg}$. The statistical distribution is further demonstrated in Table 9. The means and standard deviations of the terminal constraints are all within a small range, which reveals that the proposed phase guidance has great robustness and performance in guidance accuracy.

\section{Conclusions}

In this paper, an endoatmospheric ascent optimal guidance law within the framework of predictor-corrector algorithm is proposed. This method uses a regular perturbation method and pseudospectral collocation scheme to successfully derive the precise analytical nonlinear prediction of the ascent trajectory with arbitrary AOA, which significantly improves the computational efficiency in comparison with the traditional method. Then, an iterative scheme is used to solve the nonlinear optimal control problem around the analytical trajectory so as to provide the optimal AOA profile with high accuracy. Several nominal and Monto Carlo simulations are carried out to evaluate the performance and robustness of the proposed guidance law. The results show that this method is applicable for various cases with large difference, the consuming time at each guidance period is less than $5 \mathrm{~ms}$, and the maximum terminal error is less than $1.0 E-3 \mathrm{deg}$ for flight-path angle and $1.0 E-2 \mathrm{~m}$ for altitude even in large dispersions and uncertainties. The optimality of the proposed method is verified by comparing the AOA profiles with the optimal ones given by famous nonlinear optimal control software GPOPS-II. Conclusively, it has high guidance accuracy, strong robustness, and low computational burden, which has great potential to be applied as baseline algorithm for online guidance.

\section{Appendix}

\section{A. Appendix}

As for the following nonlinear integral initial value problem:

$$
\dot{x}=F(x, t) ; x\left(t_{0}\right)=x_{0},
$$

it is difficult to obtain the analytical solution directly. The regular perturbation technique $[43,44]$ is an effective method to derive its approximate analytic solution. Firstly, (A.1) needs be rewritten as

$$
\dot{x}=f(x, t)+\frac{\varepsilon g(x, t)}{k},
$$

where $\varepsilon$ is a small parameter for the subsequent regular perturbation order and $k$ is a constant, which is set to be equal to $\varepsilon$. According to the regular perturbation technique, $x$ can be expanded in terms of a parameter $\varepsilon$ as follows:

$$
x=x^{(0)}+\varepsilon x^{(1)}+\cdots+\varepsilon^{i} x^{(i)}+\cdots,
$$

where the superscripts $(i)$ are used to denote the ith-order solutions. Substituting (A.3) into (A.2) yields

$$
\begin{aligned}
\dot{x}^{(0)}+ & \varepsilon \dot{x}^{(1)}+\cdots+\varepsilon^{i} \dot{x}^{(i)}+\cdots \\
= & f\left(x^{(0)}+\varepsilon x^{(1)}+\cdots+\varepsilon^{i} x^{(i)}+\cdots, t\right) \\
& +\varepsilon \frac{g\left(x^{(0)}+\varepsilon x^{(1)}+\cdots+\varepsilon^{i} x^{(i)}+\cdots, t\right)}{k} .
\end{aligned}
$$

By expanding (A.4) into the Taylor series expansion and treating both sides of the equation according to the equal coefficient of $\varepsilon$ to the same power, the expanded-form dynamics can be obtained, where the zeroth-order and first-order dynamics are

$$
\begin{aligned}
& \dot{x}^{(0)}=f\left(x^{(0)}, t\right), \\
& \dot{x}^{(1)}=f_{x}\left(x^{(0)}, t\right) x^{(1)}+g\left(x^{(0)}, t\right),
\end{aligned}
$$

where $f_{x}(x, t)$ is the partial derivative of $f(x, t)$ to $x$. It can be found from (A.5) that

(1) the zero-order dynamics is only concerned with zero-order items. By selecting proper $f(x, t)$, the zero-order items can be solved analytically

(2) The first coefficient and the second term on the right side of the first-order dynamics are functions of the zero-order items. Under the premise that the analytical solution to zero-order items have been obtained, the first-order dynamics is a linear time-varying system, which can be solve analytically using some numerical technologies

Therefore, the solution of state $x$ can be approximated as (A.6) with high accuracy.

$$
x \approx x^{(0)}+\varepsilon x^{(1)}
$$

It is noted that the accuracy of analytical solution depends on the difference between $f(x, t)$ and $g(x, t)$. The more $|f(x, t)|$ is larger than $|g(x, t)|$; the higher the accuracy of the analytic solution will be, and vice versa. Therefore, the key of using regular perturbation method is how to select the appropriate functions, $f(x, t)$ and $g(x, t)$, which can not only ensure the accuracy of solution but also facilitate analytically integrating.

\section{B. Appendix}

As for the integral term $\int_{\bar{V}_{0}}^{\bar{V}} f\left(x_{\bar{V}}\right) d x_{\bar{V}}$, let

$$
f\left(x_{\bar{V}}\right) \approx \sum_{i=0}^{N} f\left(x_{\bar{V} i}\right) L_{i}(x)=\sum_{i=0}^{N} p_{i} x^{i}
$$


where $x_{\bar{V} i}$ is the value of $\bar{V}$ at the $i$-th interpolation point $x_{i}$ and $L_{i}(x)(i=0, \cdots, N)$ is the interpolation basis function defined as

$$
L_{i}(x)=\prod_{j=0, j \neq i}^{N} \frac{x-x_{j}}{x_{i}-x_{j}}
$$

where the interpolation points $x_{i}(i=0, \cdots, N) \in(-1,1)$ are the roots of Chebyshev polynomials.

$$
x_{i}=\cos \left[\frac{(2 i+1) \pi}{2 N+2}\right], \quad i=0, \cdots, N \text {. }
$$

$x=\left(x_{\bar{V}}-\bar{V}_{2}\right) / \bar{V}_{1}$ is the dimensionless variable with $[-1$, 1], where $\bar{V}_{1}=\left(\bar{V}-\bar{V}_{0}\right) / 2$ and $\bar{V}_{2}=\left(\bar{V}+\bar{V}_{0}\right) / 2$. It is obvious that $L_{i}(x)$ is a $N$-th order polynomial, which can be rewritten as

$$
L_{i}(x)=\sum_{j=0}^{N} D_{c b}^{(i, j)} x^{j}
$$

where the coefficients $D_{c b}^{(i, j)}$ are

$$
\left\{\begin{array}{l}
D_{c b}^{(N-i, 0)}=\frac{1}{\Delta_{i}}, \\
D_{c b}^{(N-i, n)}=\frac{\sum_{j=0, j \neq i}^{N-n+1}\left(x_{j} \sum_{k=j+1, k \neq i}^{N-n+2} d_{n-1, k}^{(i)}\right)}{\Delta_{i}},
\end{array}\right.
$$

where $\Delta_{i}=\prod_{j=0, j \neq i}^{N}\left(x_{i}-x_{j}\right)$ and $d_{n-1, k}^{(i)}$ meet the following recurrence relation:

$$
\left\{\begin{array}{l}
d_{0, j}^{(i)}=1, \\
d_{n, j}^{(i)}=(-1)^{n} x_{j} \sum_{k=j+1, k \neq i}^{N-n+2} d_{n-1, k}^{(i)}
\end{array}\right.
$$

Therefore, the coefficient $p_{i}(i=0, \cdots, N)$ is

$$
p_{i}=\sum_{j=0}^{N} D_{c b}^{(j, i)} f\left(x_{\bar{V} i}\right)
$$

Then, the analytical solution to $\int_{\bar{V}_{0}}^{\bar{V}} f\left(x_{\bar{V}}\right) d x_{\bar{V}}$ is derived as

$$
\int_{\bar{V}_{0}}^{\bar{V}} f\left(x_{\bar{V}}\right) d x_{\bar{V}}=\sum_{i=0}^{N} p_{i} \omega_{i}(\bar{V})
$$

where $\omega_{i}(\bar{V})$ is

$\omega_{i}(\bar{V})=\int_{\bar{V}_{0}}^{\bar{V}}\left[\frac{\left(x_{\bar{V}}-\bar{V}_{2}\right)}{\bar{V}_{1}}\right]^{i} d x_{\bar{V}}=\frac{1}{2}\left(\bar{V}-\bar{V}_{0}\right) \frac{\left[x^{i+1}-(-1)^{i+1}\right]}{(i+1)}$.

Substituting (A.13) into (A.14), $\int_{\bar{V}_{0}}^{\bar{V}} f\left(x_{\bar{V}}\right) d x_{\bar{V}}$ can be rewritten as

$$
\int_{\bar{V}_{0}}^{\bar{V}} f\left(x_{\bar{V}}\right) d x_{\bar{V}}=\frac{1}{2}\left(\bar{V}-\bar{V}_{0}\right) \sum_{i=0}^{N} W_{i}(x) f\left(x_{\bar{V} i}\right)
$$

where $W_{i}(x)$ is the integral weight coefficient expressed as

$$
W_{i}(x)=\sum_{j=0}^{N} D_{c b}^{(j, i)} \frac{\left[x^{j+1}-(-1)^{j+1}\right]}{(j+1)} .
$$

\section{Data Availability}

The simulation data used to support the findings of this study are available from the corresponding author upon request.

\section{Conflicts of Interest}

The authors declare that they have no conflicts of interest.

\section{Acknowledgments}

This work is supported in part by the National Natural Science Foundation of China (NSFC) under Grant 62003019.

\section{References}

[1] M. Sun, Y. Zhang, J. Liu, J. Huang, Z. Wang, and Z. Chen, "Explicit parameterized energy management for scramjet transition," IEEE Transactions on Aerospace and Electronic Systems, vol. 55, no. 2, pp. 798-809, 2019.

[2] X. Yue, X. Shao, and W. Zhang, "Elliptical encircling of quadrotors for a dynamic target subject to aperiodic signals updating," IEEE Transactions on Intelligent Transportation Systems, 2021.

[3] X. Shao, Y. Shi, W. Zhang, and H. Cao, "Neurodynamic approximation-based quantized control with improved transient performances for MEMS gyroscopes: theory and experimental results," IEEE Transactions on Industrial Electronics, vol. 68, no. 10, pp. 9972-9983, 2020.

[4] X. Shao and Y. Shi, "Neural-network-based constrained output-feedback control for MEMS gyroscopes considering scarce transmission bandwidth," IEEE Transactions on Cybernetics, 2021.

[5] D. E. Kirk, Optimal Control Theory: An Introduction, Prentice-Hall, Upper Saddle River, NJ, 1970.

[6] P. Lu, H. Sun, and B. Tsai, "Closed-loop endoatmospheric ascent guidance," Journal of Guidance, Control, and Dynamics, vol. 26, no. 2, pp. 283-294, 2003.

[7] O. Murillo and P. Lu, "Fast ascent trajectory optimization for hypersonic air-breathing vehicles," in AIAA Guidance, 
Navigation, and Control Conference, Toronto, Ontario, Canada, 2010

[8] P. Lu and B. Pan, "Highly constrained optimal launch ascent guidance," Journal of Guidance, Control, and Dynamics, vol. 33, no. 2, pp. 404-414, 2010.

[9] P. Lu, L. Zhang, and H. Sun, "Ascent guidance for responsive launch: a fixed-point approach," in AIAA Guidance, Navigation, and Control Conference and Exhibit, San Francisco, California, 2005.

[10] B. Lu, N. Cui, Y. Fu, W. Shan, and X. Chang, "Closed-loop atmospheric ascent guidance based on finite element method," Aircraft Engineering and Aerospace Technology, vol. 87, no. 5, pp. 393-401, 2015.

[11] G. Dukeman and A. Calise, "Enhancements to an atmospheric ascent guidance algorithm," in AIAA Guidance, Navigation, and Control Conference and Exhibit, Austin, Texas, 2003.

[12] G. Dukeman, "Atmospheric ascent guidance for rocketpowered launch vehicles," in AIAA Guidance, Navigation, and Control Conference and Exhibit, Monterey, California, 2002.

[13] X. Cheng, H. Li, and R. Zhang, "Efficient ascent trajectory optimization using convex models based on the NewtonKantorovich/pseudospectral approach," Aerospace Science and Technology, vol. 66, pp. 140-151, 2017.

[14] H. Duan and S. Li, "Artificial bee colony-based direct collocation for reentry trajectory optimization of hypersonic vehicle," IEEE Transactions on Aerospace and Electronic Systems, vol. 51, no. 1, pp. 615-626, 2015.

[15] W. Fu, B. Wang, X. Li, L. Liu, and Y. Wang, "Ascent trajectory optimization for hypersonic vehicle based on improved chicken swarm optimization," IEEE Access, vol. 7, pp. 151836-151850, 2019.

[16] M. Pontani, "Particle swarm optimization of ascent trajectories of multistage launch vehicles," Acta Astronautica, vol. 94, no. 2, pp. 852-864, 2014.

[17] R. Giri and D. Ghose, "Differential evolution based ascent phase trajectory optimization for a hypersonic vehicle," in International Conference on Swarm, Evolutionary, and Memetic Computing, Berlin, Heidelberg, 2010.

[18] H. M. Prasanna, D. Ghose, M. S. Bhat, C. Bhattacharyya, and J. Umakant, "Ascent phase trajectory optimization for a hypersonic vehicle using nonlinear programming," in International Conference on Computational Science and Its Applications, Berlin, Heidelberg, 2005.

[19] C. Song, G. Zhao, and Y. Zhi, "Boost phase trajectory optimization for hypersonic vehicle based on GPM," in $20113 \mathrm{rd}$ International Conference on Computer Research and Development, Shanghai, China, 2011.

[20] W. Shi, Z. Jing, and Y. Yang, "Ascent trajectory optimisation for hypersonic vehicles via Gauss pseudospectral method," International Journal of Space Science and Engineering, vol. 1, no. 1, pp. 64-81, 2013.

[21] L. Fei, C. Tao, W. Songyan, and Y. Ming, "Trajectory optimization for launch vehicle boost phase based on Gauss pseudospectral method," in The 35th Chinese Control Conference, pp. 10910-10914, Chengdu, China, 2016.

[22] S. Yang, T. Cui, X. Hao, and D. Yu, “Trajectory optimization for a ramjet-powered vehicle in ascent phase via the Gauss pseudospectral method," Aerospace Science and Technology, vol. 67, pp. 88-95, 2017.

[23] T. C. Z. Sun, S. Wang, and M. Yang, "Optimization method of multi-stages launch vehicle trajectory based on segmental
Chebyshev pseudo spectral method," in The 36th Chinese Control Conference, pp. 5839-5844, Dalian, China, 2017.

[24] J. Zhou, H. Lei, and D. Zhang, "Online optimal midcourse trajectory modification algorithm for hypersonic vehicle interceptions," Aerospace Science and Technology, vol. 63, pp. 266277, 2017.

[25] D. Zhang, L. Liu, and Y. Wang, "On-line ascent phase trajectory optimal guidance algorithm based on pseudospectral method and sensitivity updates," Journal of Navigation, vol. 68, no. 6, pp. 1056-1074, 2015.

[26] D. Zhang, X. Lu, L. Liu, and Y. Wang, "An online ascent phase trajectory reconstruction algorithm using Gauss pseudospectral method," in 2012 International Conference on Modelling, Identification and Control, pp. 1184-1189, Wuhan, China, 2012.

[27] D. A. Benson, G. T. Huntington, T. P. Thorvaldsen, and A. V. Rao, "Direct trajectory optimization and costate estimation via an orthogonal collocation method," Journal of Guidance, Control, and Dynamics, vol. 29, no. 6, pp. 1435-1440, 2006.

[28] M. Li, J. Hu, and H. Huang, "A segmented and weighted adaptive predictor-corrector guidance method for the ascent phase of hypersonic vehicle," Aerospace Science and Technology, vol. 106, p. 106231, 2020.

[29] L. Yang, H. Zhou, and W. Chen, "Application of linear gauss pseudospectral method in model predictive control," Acta Astronautica, vol. 96, pp. 175-187, 2014.

[30] L. Yang, J. Yang, W. Chen, and H. Liu, "Entry guidance with no-fly zone avoidance using linear pseudospectral model predictive control," IEEE Access, vol. 7, pp. 98589-98602, 2019.

[31] L. Yang, X. Liu, W. Chen, and H. Zhou, "Autonomous entry guidance using linear pseudospectral model predictive control," Aerospace Science and Technology, vol. 80, pp. 38-55, 2018.

[32] L. Yang, W. Chen, X. Liu, and H. Zhou, "Robust entry guidance using multi-segment linear pseudospectral model predictive control," Journal of Systems Engineering and Electronics, vol. 28, no. 1, pp. 103-125, 2017.

[33] W. Zhang, W. Chen, and W. Yu, "Analytical solutions to three-dimensional hypersonic gliding trajectory over rotating earth," Acta Astronautica, vol. 179, pp. 702-716, 2021.

[34] W. Yu, P. Zhao, and W. Chen, "Analytical solutions to aeroassisted orbital transfer problem," IEEE Transactions on Aerospace and Electronic Systems, vol. 56, no. 5, pp. 3502-3515, 2020.

[35] P. Zhao, W. Chen, and W. Yu, “Analytical solutions for longitudinal-plane motion of hypersonic skip-glide trajectory," Nonlinear Dynamics, vol. 96, no. 3, pp. 1947-1969, 2019.

[36] W. Yu, W. Chen, Z. Jiang, W. Zhang, and P. Zhao, “Analytical entry guidance for coordinated flight with multiple no-flyzone constraints," Aerospace Science and Technology, vol. 84, pp. 273-290, 2019.

[37] W. Yu and W. Chen, "Entry guidance with real-time planning of reference based on analytical solutions," Advances in Space Research, vol. 55, no. 9, pp. 2325-2345, 2015.

[38] P. Lu, "Analytical solutions to constrained hypersonic flight trajectories," Journal of Guidance, Control, and Dynamics, vol. 16, no. 5, pp. 956-960, 1993.

[39] S. Ann, S. Lee, Y. Kim, and J. Ahn, "Midcourse guidance for exoatmospheric interception using response surface based trajectory shaping," IEEE Transactions on Aerospace and Electronic Systems, vol. 56, no. 5, pp. 3655-3673, 2020. 
[40] S. He and C.-H. Lee, "Optimal impact angle guidance for exoatmospheric interception utilizing gravitational effect," IEEE Transactions on Aerospace and Electronic Systems, vol. 55, no. 3, pp. 1382-1392, 2019.

[41] M. A. Patterson and A. V. Rao, "GPOPS-II," ACM transactions on mathematical software, vol. 41, no. 1, pp. 1-37, 2014.

[42] D. A. Benson, A Gauss Pseudospectral Transcription for Optimal Control. [Ph.D thesis], Department of Aeronautics and Astronautics, MIT, 2004.

[43] A. H. Nayfeh, Perturbation Methods, Wiley, New York, 1973.

[44] J. Kevorkian and J. D. Cole, Perturbation Methods in Applied Mathematics, Springer, New York, 1981. 